\title{
MULTIMODELS FOR INCOMPRESSIBLE FLOWS: ITERATIVE SOLUTIONS FOR THE NAVIER-STOKES/OSEEN COUPLING*
}

\author{
L. Fatone ${ }^{1}$, P. Gervasio ${ }^{2}$ And A. Quarteroni ${ }^{1,3}$
}

\begin{abstract}
In a recent paper [4] we have proposed and analysed a suitable mathematical model which describes the coupling of the Navier-Stokes with the Oseen equations. In this paper we propose a numerical solution of the coupled problem by subdomain splitting. After a preliminary analysis, we prove a convergence result for an iterative algorithm that alternates the solution of the Navier-Stokes problem to the one of the Oseen problem.
\end{abstract}

Mathematics Subject Classification. 76D05, 65M55, 65M60,65M70.

Received: September 14, 2000. Revised: February, 2001.

\section{INTRODUCTION}

The coupling between the full Navier-Stokes equations for incompressible flows and a convenient reduced model can be based on different kind of strategies, for example dropping the viscous stresses from the momentum equations, or linearising the convective terms, or again assuming an irrotational flow regime in a subregion of the computational domain (e.g. see $[5-7,10,13])$.

In this paper we focus on the Navier-Stokes problem coupled with the linear Oseen equations (a linear approximation of the flow) via suitable transmission conditions at the interface. This problem was introduced and motivated in [3] and [4], where a well-posedness analysis has been carried out. In this paper, we propose an iterative domain decomposition method to solve the heterogeneous problem via suitable splitting of the interface continuity conditions.

More precisely, at each iteration we solve a Navier-Stokes problem in a subregion $\Omega_{1}$ of the computational domain $\Omega$ imposing the continuity of the velocity field at the interface $\Gamma$. Next, we solve the linear Oseen problem in the complementary subdomain $\Omega_{2}$; this time we enforce on $\Gamma$ the continuity of the normal stress (see Fig. 1 for two examples of domain splitting). This procedure yields a Dirichlet problem for Navier-Stokes equations in $\Omega_{1}$ and a Neumann problem for Oseen equations in $\Omega_{2}$, at each iteration. Yet, the Dirichlet/Neumann iterations can be rewritten as a preconditioned Richardson procedure for the non linear Steklov-Poincaré equation.

The convergence analysis of the proposed iterative scheme is carried out by constructing a proper fixed-point operator $T_{\theta}+G_{\theta}$, which involves a relaxation parameter $\theta$, and by proving that it is a contraction. Under

Keywords and phrases. Navier-Stokes equations, domain decomposition methods, iterative schemes, convergence analysis.

* This research has been carried out with the support of M.U.R.S.T., 1998 "Metodologie Numeriche Avanzate per il Calcolo Scientifico" and Swiss National Science Foundation Project N. 21-54139.98 .

1 Department of Mathematics, Politecnico di Milano, 20133 Milano Italy. e-mail: fatone@mate.polimi.it

2 Department of Mathematics, University of Brescia, 25100 Brescia Italy. e-mail: gervasio@ing.unibs.it

3 Department of Mathematics, EPFL, 1015 Lausanne, Switzerland. e-mail: Alfio.Quarteroni@epfl.ch 
suitable hypotheses of "smallness" of both the forcing term, the far-field velocity $\mathbf{u}_{\infty}$ (introduced in the Oseen problem) and the initial data $\boldsymbol{\lambda}^{0}$ (for the Richardson procedure) we prove that $T_{\theta}+G_{\theta}$ is a contraction.

On the ground of this convergence result we also prove existence and uniqueness of the solution to the heterogeneous Navier-Stokes/Oseen problem.

The assessment of our theoretical results on a couple of test problems is also carried out in this paper. The Kovasznay analytical solution [8] is considered to test the convergence of the iterative Dirichlet/Neumann algorithm for different values of the relaxation parameter $\theta$. With the second test case we extend the twodomains formulation to the case of many subdomains and investigate the dependence of the Dirichlet/Neumann convergence rate on the Reynolds number.

An outline of this paper is as follows: in Section 1 we recall the Navier-Stokes equations and introduce the basic notations. In Section 2 we recall the coupled Navier-Stokes/Oseen model with the interface conditions for the multidomain formulation that was introduced in [4]. In Section 3 we introduce the Dirichlet/Neumann iterative method. In Section 4 we give a-priori estimates for Navier-Stokes equations. In Section 5 we introduce the non linear Steklov-Poincaré operator. Then we prove that the Richardson method on the Steklov-Poincaré equation can be reinterpreted as a fixed-point iteration, and prove that the fixed-point map is a contraction. In Section 6, from the previous convergence proof, we deduce an existence and uniqueness result for the coupled Navier-Stokes/Oseen problem. Finally in Section 7 we report the numerical results on two test cases.

\section{The Navier-Stokes problem}

Let $\Omega$ be a Lipschitz, bounded open set in $\mathbb{R}^{d}, d=2,3$, with boundary $\partial \Omega$.

The $d$-dimensional, steady, viscous, incompressible Navier-Stokes equations with homogeneous Dirichlet boundary conditions read as follows: given $\mathbf{f}: \Omega \rightarrow \mathbb{R}^{d}$, find $\mathbf{u}: \Omega \rightarrow \mathbb{R}^{d}$ and $p: \Omega \rightarrow \mathbb{R}$ such that

$$
-\nu \Delta \mathbf{u}+(\mathbf{u} \cdot \nabla) \mathbf{u}+\nabla p=\mathbf{f}, \quad \nabla \cdot \mathbf{u}=0 \quad \text { in } \Omega,
$$

with $\mathbf{u}=\mathbf{0}$ on $\partial \Omega$, where $\mathbf{u}$ and $p$ represent, respectively, the velocity and the pressure of the fluid, and $\nu=$ const $>0$ is the viscosity coefficient.

The weak formulation is as follows: given $\mathbf{f} \in\left[H^{-1}(\Omega)\right]^{d}$, find $\mathbf{u} \in\left[H_{0}^{1}(\Omega)\right]^{d}$ and $p \in L_{0}^{2}(\Omega)$ such that

$$
\begin{cases}c(\mathbf{u} ; \mathbf{u}, \mathbf{v})+b(\mathbf{v}, p)=\mathcal{F}(\mathbf{v}) & \forall \mathbf{v} \in\left[H_{0}^{1}(\Omega)\right]^{d} \\ b(\mathbf{u}, q)=0 & \forall q \in L_{0}^{2}(\Omega),\end{cases}
$$

where the trilinear form $c:\left[H^{1}(\Omega)\right]^{d} \times\left[H^{1}(\Omega)\right]^{d} \times\left[H^{1}(\Omega)\right]^{d} \rightarrow \mathbb{R}$ and the bilinear form $b:\left[H^{1}(\Omega)\right]^{d} \times L^{2}(\Omega) \rightarrow \mathbb{R}$ are defined as follows

$$
\begin{gathered}
c(\mathbf{w} ; \mathbf{u}, \mathbf{v}):=\nu \int_{\Omega} \nabla \mathbf{u} \nabla \mathbf{v} \mathrm{d} \Omega+\int_{\Omega}(\mathbf{w} \cdot \nabla) \mathbf{u v} \mathrm{d} \Omega, \\
b(\mathbf{u}, q):=-\int_{\Omega} q \nabla \cdot \mathbf{u} \mathrm{d} \Omega .
\end{gathered}
$$

We note that if non-homogeneous Dirichlet boundary conditions on $\partial \Omega$ hold, then the functional spaces must be conveniently adapted (see [12]).

In the following sections we will use also the following linear forms: $a:\left[H^{1}(\Omega)\right]^{d} \times\left[H^{1}(\Omega)\right]^{d} \rightarrow \mathbb{R}$

$$
a(\mathbf{u}, \mathbf{v}):=\int_{\Omega} \nabla \mathbf{u} \nabla \mathbf{v} \mathrm{d} \Omega
$$



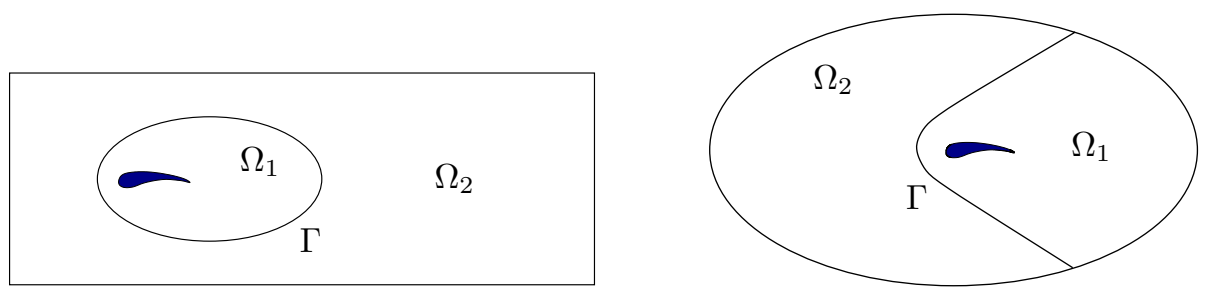

Figure 1. Two different decompositions of the computational domain $\Omega$ into a Navier-Stokes subdomain $\left(\Omega_{1}\right)$ and an Oseen subdomain $\left(\Omega_{2}\right)$.

and $e:\left[H^{1}(\Omega)\right]^{d} \times\left[H^{1}(\Omega)\right]^{d} \times\left[H^{1}(\Omega)\right]^{d} \rightarrow \mathbb{R}$

$$
e(\mathbf{w} ; \mathbf{u}, \mathbf{v}):=\int_{\Omega}(\mathbf{w} \cdot \nabla) \mathbf{u v} \mathrm{d} \Omega=\sum_{i, j=1}^{d}\left(w_{j} \frac{\partial u_{i}}{\partial x_{j}}, v_{i}\right) .
$$

Furthermore we define the linear functional $\mathcal{F}:\left[H^{1}(\Omega)\right]^{d} \rightarrow \mathbb{R}$

$$
\mathcal{F}(\mathbf{v}):=\langle\mathbf{f}, \mathbf{v}\rangle,
$$

where $\langle\cdot, \cdot\rangle$ stands for the duality pairing between $\left[H^{1}(\Omega)\right]^{d}$ and its dual space.

Under appropriate hypotheses on $\mathbf{f}$ there exists a unique solution of (2). More precisely, the solution may not be unique when $\nu$ is small compared to the external force field $\mathbf{f}$ (see [14,15]).

\section{A heterogeneous domain DeCOMPosition MOdEL}

Our coupled model is based on the following idea: in a subdomain $\Omega_{1}$ we consider the full Navier-Stokes system (1), while in a subdomain $\Omega_{2}$ we approximate the non-linear Navier-Stokes equations by the linear Oseen system:

$$
-\nu \Delta \mathbf{u}+\left(\mathbf{u}_{\infty} \cdot \nabla\right) \mathbf{u}+\nabla p=\mathbf{f}, \quad \nabla \cdot \mathbf{u}=0 \quad \text { in } \Omega_{2} .
$$

$\mathbf{u}_{\infty}$ is a prescribed solenoidal vector field that can represent, for instance, the far-field velocity at the boundary of the domain $\Omega_{2}$, or any preliminary guess for $\mathbf{u}$.

We also require that $\bar{\Omega}=\bar{\Omega}_{1} \cup \bar{\Omega}_{2}$ and we define the interface $\Gamma:=\partial \Omega_{1} \cap \partial \Omega_{2}$.

The two systems (1), (8) are then coupled at the interface $\Gamma$ via suitable transmission conditions.

Precisely, a formulation of the coupled Navier-Stokes/Oseen problem, related to (1), reads as follows: find $\mathbf{u}_{i}: \Omega_{i} \rightarrow \mathbb{R}^{d}$ and $p_{i}: \Omega_{i} \rightarrow \mathbb{R}, i=1,2$, such that

$$
\begin{array}{ll}
-\nu \Delta \mathbf{u}_{1}+\left(\mathbf{u}_{1} \cdot \nabla\right) \mathbf{u}_{1}+\nabla p_{1}=\mathbf{f}, \quad \nabla \cdot \mathbf{u}_{1}=0 & \text { in } \Omega_{1} \\
\mathbf{u}_{1}=\mathbf{u}_{2} & \text { on } \Gamma \\
\nu \frac{\partial \mathbf{u}_{1}}{\partial \mathbf{n}}-p_{1} \mathbf{n}-\frac{1}{2}\left(\mathbf{u}_{1} \cdot \mathbf{n}\right) \mathbf{u}_{1}=\nu \frac{\partial \mathbf{u}_{2}}{\partial \mathbf{n}}-p_{2} \mathbf{n}-\frac{1}{2}\left(\mathbf{u}_{\infty} \cdot \mathbf{n}\right) \mathbf{u}_{2} & \text { on } \Gamma \\
-\nu \Delta \mathbf{u}_{2}+\left(\mathbf{u}_{\infty} \cdot \nabla\right) \mathbf{u}_{2}+\nabla p_{2}=\mathbf{f}, \quad \nabla \cdot \mathbf{u}_{2}=0 & \text { in } \Omega_{2}
\end{array}
$$

and $\mathbf{u}_{i}=\mathbf{0}$ on $\partial \Omega_{i} \cap \partial \Omega, i=1,2$, where $\mathbf{u}_{i}:=\mathbf{u}_{\mid \Omega_{i}}, p_{i}:=p_{\mid \Omega_{i}}$ and $\mathbf{n}$ is the outward normal vector to $\Gamma$ directed from $\Omega_{1}$ to $\Omega_{2}$. 
The equations (10) and (11) are called the transmission conditions on the interface $\Gamma$ for problem (9)-(12). The former imposes the continuity of the velocity field, while the latter is derived from having written the convective term under the following form:

$$
((\mathbf{w} \cdot \nabla) \mathbf{u}, \mathbf{v})_{\Omega}=\frac{1}{2}((\mathbf{w} \cdot \nabla) \mathbf{u}, \mathbf{v})_{\Omega}+\frac{1}{2} \sum_{i=1}^{2}\left(\left(\mathbf{w}_{\mid \Omega_{i}} \cdot \nabla\right) \mathbf{u}_{\mid \Omega_{i}}, \mathbf{v}_{\mid \Omega_{i}}\right)_{\Omega_{i}}
$$

and having applied the Green's formula to equations (9) and (12).

Alternatively, we could consider

$$
\nu \frac{\partial \mathbf{u}_{1}}{\partial \mathbf{n}}-p_{1} \mathbf{n}=\nu \frac{\partial \mathbf{u}_{2}}{\partial \mathbf{n}}-p_{2} \mathbf{n} \quad \text { on } \Gamma
$$

instead of (11), which entails the continuity of the normal (linear) stress vector on $\Gamma$, as we did in [4].

In this paper we limit ourselves to consider the interface conditions (10) and (11). From now on we refer to (11) as the transmission condition on the Oseen flux and to (14) as the transmission condition on the Stokes flux.

A comparative analysis between these two possible choices is addressed in [4].

Feistauer and Schwab in [5] have proved that the choice of transmission conditions (10) and (11) ensure the existence of a weak solution of the coupled problem (9)-(12) for all data, also in the case of unbounded domains. In this paper we will prove the existence and the uniqueness of the weak solution of (9)-(12) for bounded domains.

For all $\mathbf{w}_{i}, \mathbf{u}_{i}, \mathbf{v}_{i} \in\left[H^{1}\left(\Omega_{i}\right)\right]^{d}$ and $q_{i} \in L^{2}\left(\Omega_{i}\right), i=1,2$, let us define the forms $a_{i}(\cdot, \cdot), b_{i}(\cdot, \cdot), c_{i}(\cdot ; \cdot, \cdot)$, $e_{i}(\cdot ; \cdot, \cdot)$ and the linear functionals $\mathcal{F}_{i}$ as being the restrictions to $\Omega_{i}$ of the forms (5), (4), (3), (6) and of the linear functional (7), respectively. Furthermore, for $i=1,2$, we introduce the forms

$$
\begin{aligned}
d_{i}\left(\mathbf{w}_{i} ; \mathbf{u}_{i}, \mathbf{v}_{i}\right): & =\nu \int_{\Omega_{i}} \nabla \mathbf{u}_{i} \nabla \mathbf{v}_{i} \mathrm{~d} \Omega+\frac{1}{2} \int_{\Omega_{i}}\left(\mathbf{w}_{i} \cdot \nabla\right) \mathbf{u}_{i} \mathbf{v}_{i} \mathrm{~d} \Omega-\frac{1}{2} \int_{\Omega_{i}}\left(\mathbf{w}_{i} \cdot \nabla\right) \mathbf{v}_{i} \mathbf{u}_{i} \mathrm{~d} \Omega \\
& =\nu a_{i}\left(\mathbf{u}_{i}, \mathbf{v}_{i}\right)+\frac{1}{2} e_{i}\left(\mathbf{w}_{i} ; \mathbf{u}_{i}, \mathbf{v}_{i}\right)-\frac{1}{2} e_{i}\left(\mathbf{w}_{i} ; \mathbf{v}_{i}, \mathbf{u}_{i}\right)
\end{aligned}
$$

where the skew-symmetric form of the convective term is highlighted.

We then define the spaces (for $i=1,2$ ):

$$
\begin{aligned}
V_{i}: & =\left\{v \in H^{1}\left(\Omega_{i}\right): v_{\mid \partial \Omega \cap \partial \Omega_{i}}=0\right\}, \quad V_{i}^{0}:=H_{0}^{1}\left(\Omega_{i}\right), \\
\Lambda & :=\left\{\mu \in H^{\frac{1}{2}}(\Gamma): \mu=v_{\mid \Gamma} \text { for a suitable } v \in H_{0}^{1}(\Omega)\right\}
\end{aligned}
$$

and we observe that

$$
d_{i}\left(\mathbf{w}_{i} ; \mathbf{u}_{i}, \mathbf{v}_{i}\right)=c_{i}\left(\mathbf{w}_{i} ; \mathbf{u}_{i}, \mathbf{v}_{i}\right)-\frac{1}{2}\left(\left(\mathbf{w}_{i} \cdot \mathbf{n}\right) \mathbf{u}_{i}, \mathbf{v}_{i}\right)_{\Gamma} \quad \forall \mathbf{v}_{i} \in\left[V_{i}\right]^{d}
$$


The weak formulation of problem (9)-(12) reads: find $\mathbf{u}_{i} \in\left[V_{i}\right]^{d}$ and $p_{i} \in L^{2}\left(\Omega_{i}\right)$, for $i=1,2$, satisfying

$$
\begin{cases}d_{1}\left(\mathbf{u}_{1} ; \mathbf{u}_{1}, \mathbf{v}_{1}\right)+b_{1}\left(\mathbf{v}_{1}, p_{1}\right)=\mathcal{F}_{1}\left(\mathbf{v}_{1}\right) & \forall \mathbf{v}_{1} \in\left[V_{1}^{0}\right]^{d} \\ b_{1}\left(\mathbf{u}_{1}, q_{1}\right)=0 & \forall q_{1} \in L_{0}^{2}\left(\Omega_{1}\right) \\ \mathbf{u}_{1}=\mathbf{u}_{2} & \text { on } \Gamma \\ d_{2}\left(\mathbf{u}_{\infty} ; \mathbf{u}_{2}, \mathbf{v}_{2}\right)+b_{2}\left(\mathbf{v}_{2}, p_{2}\right)=\mathcal{F}_{2}\left(\mathbf{v}_{2}\right) & \forall \mathbf{v}_{2} \in\left[V_{2}^{0}\right]^{d} \\ b_{2}\left(\mathbf{u}_{2}, q_{2}\right)=0 & \forall q_{2} \in L^{2}\left(\Omega_{2}\right) \\ d_{2}\left(\mathbf{u}_{\infty} ; \mathbf{u}_{2}, \boldsymbol{\mathcal { R }}_{2} \boldsymbol{\mu}\right)+b_{2}\left(\boldsymbol{\mathcal { R }}_{2} \boldsymbol{\mu}, p_{2}\right)=\mathcal{F}_{1}\left(\mathcal{R}_{1} \boldsymbol{\mu}\right)+\mathcal{F}_{2}\left(\mathcal{R}_{2} \boldsymbol{\mu}\right) & \\ \quad-d_{1}\left(\mathbf{u}_{1} ; \mathbf{u}_{1}, \mathcal{R}_{1} \boldsymbol{\mu}\right)-b_{1}\left(\mathcal{R}_{1} \boldsymbol{\mu}, p_{1}\right) & \forall \boldsymbol{\mu} \in[\Lambda]^{d} \\ \int_{\Omega_{1}} p_{1} \mathrm{~d} \Omega+\int_{\Omega_{2}} p_{2} \mathrm{~d} \Omega=0, & \end{cases}
$$

where $\boldsymbol{\mathcal { R }}_{i}$ indicates any linear and continuous extension operator from $[\Lambda]^{d}$ to $\left[V_{i}\right]^{d}$.

\section{A Dirichlet/Neumann iterative method}

Let

$$
\widehat{\boldsymbol{\Lambda}}:=\left\{\boldsymbol{\mu} \in[\Lambda]^{d}: \int_{\Gamma} \boldsymbol{\mu} \cdot \mathbf{n d} \gamma=0\right\}
$$

be the trace space on $\Gamma$ of divergence-free functions belonging to $\left[H_{0}^{1}(\Omega)\right]^{d}$, endowed with the norm $\|\cdot\|_{\Lambda}$ of $[\Lambda]^{d}$.

The iterative method that we propose to decouple problem (16) reads as follows. Given $\boldsymbol{\lambda}^{0} \in \widehat{\boldsymbol{\Lambda}}$, for all $k \geq 1$ :

$$
\begin{cases}\text { find }\left(\mathbf{u}_{1}^{k}, p_{1}^{k}\right) \in\left[V_{1}\right]^{d} \times L^{2}\left(\Omega_{1}\right): & \\ d_{1}\left(\mathbf{u}_{1}^{k} ; \mathbf{u}_{1}^{k}, \mathbf{v}_{1}\right)+b_{1}\left(\mathbf{v}_{1}, p_{1}^{k}\right)=\mathcal{F}_{1}\left(\mathbf{v}_{1}\right) & \forall \mathbf{v}_{1} \in\left[V_{1}^{0}\right]^{d} \\ b_{1}\left(\mathbf{u}_{1}^{k}, q_{1}\right)=0 & \forall q_{1} \in L_{0}^{2}\left(\Omega_{1}\right) \\ \mathbf{u}_{1}^{k}=\boldsymbol{\lambda}^{k-1} & \text { on } \Gamma \\ \int_{\Omega_{1}} p_{1}^{k} \mathrm{~d} \Omega+\int_{\Omega_{2}} p_{2}^{k-1} \mathrm{~d} \Omega=0, & \end{cases}
$$

then

$$
\left\{\begin{aligned}
\text { find }\left(\mathbf{u}_{2}^{k}, p_{2}^{k}\right) \in\left[V_{2}\right]^{d} \times L^{2}\left(\Omega_{2}\right): & \\
d_{2}\left(\mathbf{u}_{\infty} ; \mathbf{u}_{2}^{k}, \mathbf{v}_{2}\right)+b_{2}\left(\mathbf{v}_{2}, p_{2}^{k}\right)=\mathcal{F}_{2}\left(\mathbf{v}_{2}\right) & \forall \mathbf{v}_{2} \in\left[V_{2}^{0}\right]^{d} \\
b_{2}\left(\mathbf{u}_{2}^{k}, q_{2}\right)=0 & \forall q_{2} \in L^{2}\left(\Omega_{2}\right) \\
d_{2}\left(\mathbf{u}_{\infty} ; \mathbf{u}_{2}^{k}, \mathcal{R}_{2} \boldsymbol{\mu}\right)+b_{2}\left(\mathcal{R}_{2} \boldsymbol{\mu}, p_{2}^{k}\right)=\mathcal{F}_{1}\left(\mathcal{R}_{1} \boldsymbol{\mu}\right)+\mathcal{F}_{2}\left(\mathcal{R}_{2} \boldsymbol{\mu}\right) & \\
-d_{1}\left(\mathbf{u}_{1}^{k} ; \mathbf{u}_{1}^{k}, \mathcal{R}_{1} \boldsymbol{\mu}\right)-b_{1}\left(\mathcal{R}_{1} \boldsymbol{\mu}, p_{1}^{k}\right) & \forall \boldsymbol{\mu} \in[\Lambda]^{d}
\end{aligned}\right.
$$

and, for $k \geq 1$, the interface value is updated as follows:

$$
\boldsymbol{\lambda}^{k}:=\theta \mathbf{u}_{2 \mid \Gamma}^{k}+(1-\theta) \boldsymbol{\lambda}^{k-1} \quad \text { on } \Gamma
$$

$\theta$ is a positive relaxation parameter that will be determined in order to ensure, and possibly to accelerate, the convergence of the iterative scheme.

Remark 3.1. A "parallel" version of the previous iterative scheme is obtained replacing by $\mathbf{u}_{1}^{k}$ with $\mathbf{u}_{1}^{k-1}$ and $p_{1}^{k}$ with $p_{1}^{k-1}$ in the last set of equations (19). 
Following a terminology introduced for elliptic problems in [2] and then generalized in [12] to the case of any second order boundary value problem, the iterative scheme (18)-(20) is called Dirichlet/Neumann method. Actually, it demands for the solution of the Navier-Stokes equations in $\Omega_{1}$ with Dirichlet boundary conditions for $\mathbf{u}_{1}$ on $\Gamma$, and the solution of the Oseen equations in $\Omega_{2}$ with natural Neumann conditions on the normal stress on $\Gamma$.

The proof of convergence of the iterative procedure (18)-(20) will be carried out in Section 5 . For that, several preliminary results on both Navier-Stokes and Oseen problems are needed: they are proven in the next section.

\section{A priori estimates for Navier-Stokes And Oseen Equations}

In this section we denote by $\Gamma$ a portion of positive measure of the boundary of a domain $\Omega$ (that plays the role of $\Omega_{1}$ or $\Omega_{2}$ ), i.e. $\Gamma \subset \partial \Omega$, and let $\mathbf{n}$ be the unit outward normal vector on $\partial \Omega$.

Moreover, instead of $\widehat{\Lambda}$ we make use of the space

$$
\widetilde{\boldsymbol{\Lambda}}:=\left\{\boldsymbol{\mu} \in[\widetilde{\Lambda}]^{d}: \int_{\Gamma} \boldsymbol{\mu} \cdot \mathbf{n} \mathrm{d} \gamma=0\right\}
$$

with

$$
\widetilde{\Lambda}:=\left\{\mu \in H^{1 / 2}(\Gamma): \mu=v_{\mid \Gamma} \text { for a suitable } v \in H^{1}(\Omega), v_{\mid \partial \Omega \backslash \Gamma}=0\right\}
$$

Also the space $\widetilde{\boldsymbol{\Lambda}}$ is endowed with the norm $\|\cdot\|_{\Lambda}$ of $[\Lambda]^{d}$.

For any $\boldsymbol{\psi} \in \widetilde{\boldsymbol{\Lambda}}$, we denote by $(\mathcal{V} \boldsymbol{\psi}, \Pi \psi) \in\left[H^{1}(\Omega)\right]^{d} \times L_{0}^{2}(\Omega)$ the solutions of the following non-linear boundary value problem:

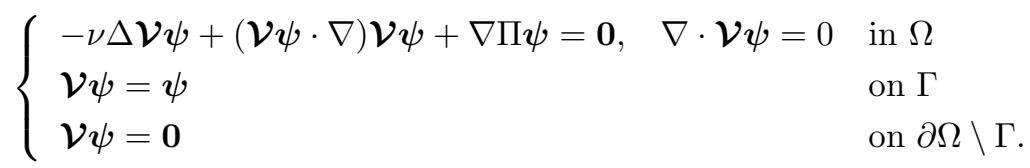

We use the abridged notation $\mathcal{V} \boldsymbol{\psi}$ instead of $\mathcal{V}(\boldsymbol{\psi})$ (the latter would be more correct, being the problem (21) non-linear). The couple $(\mathcal{V} \psi, \Pi \psi)$ is called Navier-Stokes extension of $\boldsymbol{\psi}$ to $\Omega$. We note that the solution $\mathcal{V} \boldsymbol{\varphi}$ of problem (21) exists and is unique under the following assumption (see [14, p. 178])

$$
|e(\mathbf{v} ; \mathcal{V} \psi, \mathbf{v})| \leq \frac{\nu}{2 C_{0}^{2}}\|\mathbf{v}\|_{1, \Omega}^{2}, \quad \forall \mathbf{v} \in\left[H^{1}(\Omega)\right]^{d}: \nabla \cdot \mathbf{v}=0
$$

For the sake of clarity, sometimes we prefer using the differential form of the equations, even though the use of the weak form is always understood for our analysis.

The next lemmas hold.

Lemma 4.1. If the norm of $\boldsymbol{\psi}$ in $\widetilde{\boldsymbol{\Lambda}}$ is sufficiently small with respect to the viscosity $\nu$, precisely

$$
\|\boldsymbol{\psi}\|_{\Lambda} \leq \zeta_{\nu}:=\frac{\nu}{3 C_{0}^{2} C_{1} C_{2}}
$$

where the constants $C_{0}=C_{0}(\Omega), C_{1}=C_{1}(\Omega)$ and $C_{2}=C_{2}(d)$ will be introduced along the proof, then the following estimate holds

$$
\|\mathcal{V} \boldsymbol{\psi}\|_{1, \Omega} \leq C_{\alpha}\|\boldsymbol{\psi}\|_{\Lambda}
$$

where $C_{\alpha}$ is a suitable positive constant depending on $\Omega$. 
Proof. Let us consider the Stokes extension of $\psi \in \widetilde{\Lambda}$ to $\Omega$, namely the solution $(\mathbf{U} \psi, R \psi)$ of the following non-homogeneous Stokes problem:

$$
\begin{cases}-\nu \Delta \mathbf{U} \boldsymbol{\psi}+\nabla R \boldsymbol{\psi}=\mathbf{0} & \text { in } \Omega \\ \nabla \cdot \mathbf{U} \boldsymbol{\psi}=0 & \text { in } \Omega \\ \mathbf{U} \boldsymbol{\psi}=\boldsymbol{\psi} & \text { on } \Gamma \\ \mathbf{U} \boldsymbol{\psi}=\mathbf{0} & \text { on } \partial \Omega \backslash \Gamma .\end{cases}
$$

Owing to Poincaré's inequality, $\exists C_{0}=C_{0}(\Omega)>1$ such that

$$
\|\mathbf{z}\|_{1, \Omega} \leq C_{0}\|\nabla \mathbf{z}\|_{0, \Omega} .
$$

Moreover, by the trace theorem for $H^{1}(\Omega), \exists C_{1}=C_{1}(\Omega)$ such that the following estimate holds:

$$
\|\mathbf{U} \boldsymbol{\psi}\|_{1, \Omega} \leq C_{1}\|\boldsymbol{\psi}\|_{\Lambda} \quad \forall \boldsymbol{\psi} \in \widetilde{\boldsymbol{\Lambda}}
$$

By subtracting (25) from (21) and setting $\mathbf{z}:=\mathcal{V} \psi-\mathbf{U} \boldsymbol{\psi}, \quad r:=\Pi \boldsymbol{\psi}-R \boldsymbol{\psi}$, we obtain

$$
\begin{cases}-\nu \Delta \mathbf{z}+(\mathcal{V} \psi \cdot \nabla) \mathcal{V} \psi+\nabla r=\mathbf{0} & \text { in } \Omega \\ \nabla \cdot \mathbf{z}=0 & \text { in } \Omega \\ \mathbf{z}=\mathbf{0} & \text { on } \partial \Omega\end{cases}
$$

Replacing $\mathcal{V} \boldsymbol{\psi}$ with $\mathbf{z}+\boldsymbol{U} \boldsymbol{\psi}$ in the momentum equation of (28), we deduce

$$
\begin{cases}-\nu \Delta \mathbf{z}+(\mathbf{z} \cdot \nabla) \mathbf{z}+(\mathbf{z} \cdot \nabla) \mathbf{U} \boldsymbol{\psi}+(\mathbf{U} \boldsymbol{\psi} \cdot \nabla) \mathbf{z}+(\mathbf{U} \boldsymbol{\psi} \cdot \nabla) \mathbf{U} \boldsymbol{\psi}+\nabla r=\mathbf{0} & \text { in } \Omega \\ \nabla \cdot \mathbf{z}=0 & \text { in } \Omega \\ \mathbf{z}=\mathbf{0} & \text { on } \partial \Omega\end{cases}
$$

The corresponding weak form is: find $\mathbf{z} \in\left[H_{0}^{1}(\Omega)\right]^{d}$ and $r \in L_{0}^{2}(\Omega)$ such that

$$
\begin{cases}\nu a(\mathbf{z}, \mathbf{v})+e(\mathbf{z} ; \mathbf{z}, \mathbf{v})+e(\mathbf{z} ; \mathbf{U} \boldsymbol{\psi}, \mathbf{v})+e(\mathbf{U} \boldsymbol{\psi} ; \mathbf{z}, \mathbf{v})+e(\mathbf{U} \boldsymbol{\psi} ; \mathbf{U} \boldsymbol{\psi}, \mathbf{v})+b(\mathbf{v}, r)=0 & \forall \mathbf{v} \in\left[H_{0}^{1}(\Omega)\right]^{d} \\ b(\mathbf{z}, q)=0 & \forall q \in L_{0}^{2}(\Omega),\end{cases}
$$

where $a(\cdot, \cdot), b(\cdot, \cdot)$ and $e(\cdot ; \cdot, \cdot)$ are defined in (5), (4) and (6), respectively.

Note that

$$
e(\mathbf{w} ; \mathbf{v}, \mathbf{v})=0 \quad \text { for any } \mathbf{w} \in\left[H^{1}(\Omega)\right]^{d}: \nabla \cdot \mathbf{w}=0 \quad \text { and } \mathbf{v} \in\left[H_{0}^{1}(\Omega)\right]^{d} .
$$

Then, taking $\mathbf{v}=\mathbf{z} \in\left[H_{0}^{1}(\Omega)\right]^{d}$ as test function, from (29) we obtain

$$
\nu a(\mathbf{z}, \mathbf{z})+e(\mathbf{z} ; \mathbf{U} \boldsymbol{\psi}, \mathbf{z})+e(\mathbf{U} \boldsymbol{\psi} ; \mathbf{U} \boldsymbol{\psi}, \mathbf{z})=0 .
$$

The trilinear form $e$ is continuous (see [15]), i.e. $\exists C_{2}=C_{2}(d, \Omega)>0$ such that

$$
|e(\mathbf{w} ; \mathbf{u}, \mathbf{v})| \leq C_{2}\|\mathbf{w}\|_{1, \Omega}\|\mathbf{u}\|_{1, \Omega}\|\mathbf{v}\|_{1, \Omega} \quad \forall \mathbf{w}, \mathbf{u}, \mathbf{v} \in\left[H^{1}(\Omega)\right]^{d},
$$

provided that $\Omega$ is an open bounded domain.

By (26) we have

$$
\frac{\nu}{C_{0}^{2}}\|\mathbf{z}\|_{1, \Omega}^{2} \leq C_{2}\|\mathbf{z}\|_{1, \Omega}\|\mathbf{U} \boldsymbol{\psi}\|_{1, \Omega}\left(\|\mathbf{z}\|_{1, \Omega}+\|\mathbf{U} \boldsymbol{\psi}\|_{1, \Omega}\right)
$$


and, owing to (27) and assumption (23) we can conclude that

$$
\|\mathbf{z}\|_{1, \Omega} \leq \frac{C_{1}}{2}\|\boldsymbol{\psi}\|_{\Lambda}
$$

The result (24), with $C_{\alpha}=3 C_{1} / 2$, follows applying the definition of $\mathbf{z}$ and (27).

Remark 4.2. We point out that hypothesis (23), which requires a limitation on the Reynolds number, is coherent with those that ensure existence and uniqueness of the solution of a general non-homogeneous NavierStokes problem (see $[14,15])$. In particular it is easy to see that (23) implies $(22)$.

The next result we want to prove concerns the solution $(\mathbf{Z}, W) \in\left[H_{0}^{1}(\Omega)\right]^{d} \times L_{0}^{2}(\Omega)$, of the following generalised Stokes problem:

$$
\begin{cases}-\nu \Delta \mathbf{Z}+(\mathbf{Z} \cdot \nabla) \mathcal{V} \varphi+(\mathcal{V} \psi \cdot \nabla) \mathbf{Z}+\nabla W=\mathbf{f} & \text { in } \Omega \\ \nabla \cdot \mathbf{Z}=0 & \text { in } \Omega \\ \mathbf{Z}=\mathbf{0} & \text { on } \partial \Omega\end{cases}
$$

where $\varphi, \psi \in \widetilde{\boldsymbol{\Lambda}}$ and $\mathcal{V} \varphi$ and $\mathcal{V} \psi$ are the first components of their Navier-Stokes extensions to $\Omega$.

We note that if $\mathcal{V} \boldsymbol{\varphi}$ satisfies an hypothesis like (22) and $\|\mathbf{f}\|_{-1, \Omega}$ is small enough with respect to the viscosity $\nu[14$, p. 178] then problem (32) has a unique solution. Same conclusion holds for problems (33) and (36) as well.

Lemma 4.3. If $\boldsymbol{\varphi}$ in $\widetilde{\boldsymbol{\Lambda}}$ satisfies the same assumption as $\boldsymbol{\psi}$ in (23), then there exists a positive constant $C_{\alpha}^{*}$ depending on $\nu$ and $\Omega$, such that

$$
\|\mathbf{Z}\|_{1, \Omega} \leq C_{\alpha}^{*}\|\mathbf{f}\|_{-1, \Omega},
$$

where $\|\cdot\|_{-1, \Omega}$ denotes the norm in $\left[H^{-1}(\Omega)\right]^{d}$.

Proof. From the weak formulation of (32) we obtain

$$
\nu\|\nabla \mathbf{Z}\|_{0, \Omega}^{2}=-e(\mathbf{Z} ; \mathcal{V} \varphi, \mathbf{Z})+\langle\mathbf{f}, \mathbf{Z}\rangle .
$$

By (31) it follows that

$$
\nu\|\nabla \mathbf{Z}\|_{0, \Omega}^{2} \leq C_{2}\|\mathbf{Z}\|_{1, \Omega}^{2}\|\mathcal{V} \varphi\|_{1, \Omega}+\|\mathbf{f}\|_{-1, \Omega}\|\mathbf{Z}\|_{1, \Omega} .
$$

The Poincaré inequality (26) and Lemma 4.1 (applied to $\varphi$ ) now yield

$$
\frac{\nu}{C_{0}^{2}}\|\mathbf{Z}\|_{1, \Omega}^{2} \leq C_{2} C_{\alpha}\|\mathbf{Z}\|_{1, \Omega}^{2}\|\varphi\|_{\Lambda}+\|\mathbf{f}\|_{-1, \Omega}\|\mathbf{Z}\|_{1, \Omega}
$$

Recalling that $C_{\alpha}=3 C_{1} / 2$ and using (23) the thesis holds true with $C_{\alpha}^{*}=\frac{2 C_{0}^{2}}{\nu}$.

We are going to prove the following results.

Lemma 4.4. Given $\boldsymbol{\psi}, \boldsymbol{\varphi} \in \widetilde{\boldsymbol{\Lambda}}$ both satisfying hypothesis (23), if we denote by $(\mathcal{V} \boldsymbol{\psi}, \Pi \boldsymbol{\psi})$ and $(\mathcal{V} \boldsymbol{\varphi}, \Pi \boldsymbol{\varphi})$ their Navier-Stokes extensions to $\Omega$, then there exists a positive constant $C_{\gamma}$ depending on $\Omega$, such that

$$
\|\mathcal{V} \varphi-\mathcal{V} \psi\|_{1, \Omega} \leq C_{\gamma}\|\varphi-\psi\|_{\Lambda}
$$


Proof. Setting

$$
\mathbf{z}:=\mathcal{V} \varphi-\mathcal{V} \psi \quad w:=\Pi \varphi-\Pi \psi
$$

and taking the difference between system (21) and the analogous system with $\varphi$ on $\Gamma$, we have

$$
\begin{cases}-\nu \Delta \mathbf{z}+(\mathbf{z} \cdot \nabla) \mathcal{V} \boldsymbol{\varphi}+(\mathcal{V} \boldsymbol{\psi} \cdot \nabla) \mathbf{z}+\nabla w=\mathbf{0} & \text { in } \Omega \\ \nabla \cdot \mathbf{z}=0 & \text { in } \Omega \\ \mathbf{z}=\boldsymbol{\varphi}-\boldsymbol{\psi} & \text { on } \Gamma \\ \mathbf{z}=\mathbf{0} & \text { on } \partial \Omega \backslash \Gamma .\end{cases}
$$

Now, we consider the Stokes extension of $(\boldsymbol{\varphi}-\boldsymbol{\psi}) \in \widetilde{\boldsymbol{\Lambda}}$ to $\Omega$, namely the solution $(\boldsymbol{U}, R)$ of the problem

$$
\begin{cases}-\nu \Delta \boldsymbol{U}+\nabla R=\mathbf{0} & \text { in } \Omega \\ \nabla \cdot \boldsymbol{U}=0 & \text { in } \Omega \\ \boldsymbol{U}=\boldsymbol{\varphi}-\boldsymbol{\psi} & \text { on } \Gamma \\ \boldsymbol{U}=\mathbf{0} & \text { on } \partial \Omega \backslash \Gamma\end{cases}
$$

If we define $\mathbf{Z}:=\mathbf{z}-\mathbf{U}, W:=w-R$, and subtract (34) from (33), we obtain

$$
\begin{cases}-\nu \Delta \mathbf{Z}+(\mathbf{Z} \cdot \nabla) \mathcal{V} \boldsymbol{\varphi}+(\mathcal{V} \boldsymbol{\psi} \cdot \nabla) \mathbf{Z}+\nabla W=-(\boldsymbol{U} \cdot \nabla) \mathcal{V} \boldsymbol{\varphi}-(\mathcal{V} \boldsymbol{\psi} \cdot \nabla) \mathbf{U} & \text { in } \Omega \\ \nabla \cdot \mathbf{Z}=0 & \text { in } \Omega \\ \mathbf{Z}=\mathbf{0} & \text { on } \partial \Omega\end{cases}
$$

This is a special instance of (32) where

$$
\mathbf{f}=-(\boldsymbol{U} \cdot \nabla) \mathcal{V} \boldsymbol{\varphi}-(\mathcal{V} \boldsymbol{\psi} \cdot \nabla) \mathbf{U}
$$

Then, Lemma 4.3 yields

$$
\|\mathbf{z}-\mathbf{U}\|_{1, \Omega} \leq C_{\alpha}^{*}\|(\boldsymbol{U} \cdot \nabla) \mathcal{V} \boldsymbol{\varphi}+(\mathcal{V} \psi \cdot \nabla) \mathbf{U}\|_{-1, \Omega}
$$

Applying Lemma 4.1 and the inequalities (27) and (31) it follows

$$
\begin{aligned}
\|(\boldsymbol{U} \cdot \nabla) \mathcal{V} \boldsymbol{\varphi}+(\mathcal{V} \boldsymbol{\psi} \nabla) \mathbf{U}\|_{-1, \Omega} & :=\sup _{\substack{\mathbf{v} \in\left[H_{0}^{1}(\Omega)\right]^{d} \\
\|\mathbf{v}\|_{1, \Omega}=1}}\left|\int_{\Omega}(\mathbf{U} \cdot \nabla) \mathcal{V} \boldsymbol{\varphi} \mathbf{v} \mathrm{d} \Omega+\int_{\Omega}(\mathcal{V} \boldsymbol{\psi} \nabla) \mathbf{U} \mathbf{v} \mathrm{d} \Omega\right| \\
& \leq C_{2}\|\mathbf{U}\|_{1, \Omega}\left[\|\mathcal{V} \boldsymbol{\varphi}\|_{1, \Omega}+\|\mathcal{V} \boldsymbol{\psi}\|_{1, \Omega}\right] \\
& \leq C_{1} C_{2} C_{\alpha}\|\boldsymbol{\varphi}-\boldsymbol{\psi}\|_{\Lambda}\left[\|\boldsymbol{\varphi}\|_{\Lambda}+\|\boldsymbol{\psi}\|_{\Lambda}\right] \\
& \leq \frac{\nu C_{1}}{C_{0}^{2}}\|\boldsymbol{\varphi}-\boldsymbol{\psi}\|_{\Lambda} .
\end{aligned}
$$

The result now follows from the triangle inequality

$$
\|\mathcal{V} \varphi-\mathcal{V} \psi\|_{1, \Omega} \leq\|\mathbf{z}-\mathbf{U}\|_{1, \Omega}+\|\mathbf{U}\|_{1, \Omega}
$$

and from (27), with $C_{\gamma}=3 C_{1}$. 
Now, let us represent the solution $(\mathbf{u}, p)$ of the Navier-Stokes problem in $\Omega$ with forcing term $\mathbf{f}$ and Dirichlet boundary data $\boldsymbol{\psi}$ on $\Gamma$ as being the sum of two terms:

$$
\mathbf{u}=\mathcal{V} \psi+\mathcal{V}^{*} \boldsymbol{\psi}, \quad p=\Pi \psi+\Pi^{*} \boldsymbol{\psi}
$$

where the couple $\left(\mathcal{V}^{*} \boldsymbol{\psi}, \Pi^{*} \boldsymbol{\psi}\right) \in\left[H_{0}^{1}(\Omega)\right]^{d} \times L_{0}^{2}(\Omega)$ is the solution of the following Navier-Stokes problem in $\Omega$ with homogeneous Dirichlet boundary data on $\partial \Omega$ :

$$
\begin{cases}-\nu \Delta \mathcal{V}^{*} \boldsymbol{\psi}+\left(\mathcal{V}^{*} \boldsymbol{\psi} \cdot \nabla\right) \mathcal{V}^{*} \boldsymbol{\psi}+\nabla \Pi^{*} \boldsymbol{\psi}+\left(\mathcal{V}^{*} \boldsymbol{\psi} \cdot \nabla\right) \mathcal{V} \boldsymbol{\psi}+(\mathcal{V} \boldsymbol{\psi} \cdot \nabla) \mathcal{V}^{*} \boldsymbol{\psi}=\mathbf{f} & \text { in } \Omega \\ \nabla \cdot \mathcal{V}^{*} \boldsymbol{\psi}=0 & \text { in } \Omega \\ \mathcal{V}^{*} \boldsymbol{\psi}=\mathbf{0} & \text { on } \partial \Omega\end{cases}
$$

We prefer using the shorthand notation $\left(\mathcal{V}^{*} \boldsymbol{\psi}, \Pi^{*} \boldsymbol{\psi}\right)$ instead of the formally correct one $\left(\mathcal{V}^{*}(\boldsymbol{\psi}, \mathbf{f}), \Pi^{*}(\boldsymbol{\psi}, \mathbf{f})\right)$.

Lemma 4.5. For all $\boldsymbol{\psi} \in \widetilde{\boldsymbol{\Lambda}}$ satisfying the assumption (23),

$$
\left\|\mathcal{V}^{*} \boldsymbol{\psi}\right\|_{1, \Omega} \leq C_{\alpha}^{*}\|\mathbf{f}\|_{-1, \Omega},
$$

where $C_{\alpha}^{*}$ is the constant introduced in Lemma 4.3.

Proof. The proof is similar to that of Lemma 4.3 and makes use of (30).

Lemma 4.6. If

$$
\nu^{2}>4 C_{0}^{2} C_{2}\|\mathbf{f}\|_{-1, \Omega},
$$

then there exists a positive constant $C_{\gamma}^{*}$, depending on $\nu, \Omega, d$ and $\mathbf{f}$, such that

$$
\left\|\mathcal{V}^{*} \boldsymbol{\varphi}-\mathcal{V}^{*} \boldsymbol{\psi}\right\|_{1, \Omega} \leq C_{\gamma}^{*}\|\boldsymbol{\varphi}-\boldsymbol{\psi}\|_{\Lambda}
$$

for all $\boldsymbol{\varphi}, \boldsymbol{\psi} \in \widetilde{\boldsymbol{\Lambda}}$ verifying the assumption (23).

Proof. Upon writing (36) in weak form, by subtraction we obtain

$$
\begin{aligned}
& \nu a\left(\mathcal{V}^{*} \boldsymbol{\varphi}-\mathcal{V}^{*} \boldsymbol{\psi}, \mathbf{v}\right)+e\left(\mathcal{V}^{*} \boldsymbol{\varphi} ; \mathcal{V} \boldsymbol{\varphi}, \mathbf{v}\right)+e\left(\mathcal{V} \boldsymbol{\varphi} ; \mathcal{V}^{*} \boldsymbol{\varphi}, \mathbf{v}\right) \\
& +e\left(\mathcal{V}^{*} \boldsymbol{\varphi} ; \mathcal{V}^{*} \boldsymbol{\varphi}, \mathbf{v}\right)-e\left(\mathcal{V}^{*} \boldsymbol{\psi} ; \mathcal{V}^{*} \boldsymbol{\psi}, \mathbf{v}\right)-e\left(\mathcal{V} \boldsymbol{\psi} \mathcal{V}^{*} \boldsymbol{\psi}, \mathbf{v}\right)-e\left(\mathcal{V}^{*} \boldsymbol{\psi} ; \mathcal{V}^{*} \boldsymbol{\psi}, \mathbf{v}\right) \\
& +b\left(\mathbf{v}, \Pi^{*} \boldsymbol{\varphi}-\Pi^{*} \boldsymbol{\psi}\right)=0 \quad \forall \mathbf{v} \in\left[H_{0}^{1}(\Omega)\right]^{d}, \\
& b\left(\mathcal{V}^{*} \boldsymbol{\varphi}-\mathcal{V}^{*} \boldsymbol{\psi}, q\right)=0 \quad \forall q \in L_{0}^{2}(\Omega), \\
& \mathcal{V}^{*} \varphi=\mathcal{V}^{*} \psi=0 \\
& \text { on } \Gamma \text {. }
\end{aligned}
$$

Adding and subtracting the terms $e\left(\mathcal{V}^{*} \boldsymbol{\varphi} ; \mathcal{V} \boldsymbol{\psi}, \mathbf{v}\right)+e\left(\mathcal{V} \boldsymbol{\mathcal { V }} ; \mathcal{V}^{*} \boldsymbol{\varphi}, \mathbf{v}\right)+e\left(\mathcal{V}^{*} \boldsymbol{\psi} ; \mathcal{V}^{*} \boldsymbol{\varphi}, \mathbf{v}\right)$ and by taking $\mathbf{v}=\mathcal{V}^{*} \boldsymbol{\varphi}-$ $\mathcal{V}^{*} \boldsymbol{\psi}$ as test function, from (39) we obtain

$$
\begin{aligned}
& \nu a\left(\mathcal{V}^{*} \boldsymbol{\varphi}-\mathcal{V}^{*} \boldsymbol{\psi}, \mathcal{V}^{*} \boldsymbol{\varphi}-\mathcal{V}^{*} \boldsymbol{\psi}\right)+e\left(\mathcal{V}^{*} \boldsymbol{\varphi}-\mathcal{V}^{*} \boldsymbol{\psi} ; \mathcal{V}^{*} \boldsymbol{\varphi}, \mathcal{V}^{*} \boldsymbol{\varphi}-\mathcal{V}^{*} \boldsymbol{\psi}\right) \\
& =-e\left(\mathcal{V}^{*} \boldsymbol{\varphi} ; \mathcal{V} \boldsymbol{\varphi}-\mathcal{V} \boldsymbol{\psi}, \mathcal{V}^{*} \boldsymbol{\varphi}-\mathcal{V}^{*} \psi\right)-e\left(\mathcal{V}^{*} \boldsymbol{\varphi}-\mathcal{V}^{*} \boldsymbol{\psi} ; \mathcal{V} \boldsymbol{\psi}, \mathcal{V}^{*} \boldsymbol{\varphi}-\mathcal{V}^{*} \boldsymbol{\psi}\right) \\
& -e\left(\mathcal{V} \varphi-\mathcal{V} \psi ; \mathcal{V}^{*} \varphi, \mathcal{V}^{*} \varphi-\mathcal{V}^{*} \psi\right)
\end{aligned}
$$


Applying the coercivity of $a(\cdot, \cdot)$ and Poincaré's inequality (26), we deduce that

$$
\begin{aligned}
\frac{\nu}{C_{0}^{2}}\left\|\mathcal{V}^{*} \boldsymbol{\varphi}-\mathcal{V}^{*} \boldsymbol{\psi}\right\|_{1, \Omega}^{2} \leq & C_{2}\left\|\mathcal{V}^{*} \boldsymbol{\varphi}-\mathcal{V}^{*} \boldsymbol{\psi}\right\|_{1, \Omega}^{2}\left\|\mathcal{V}^{*} \boldsymbol{\varphi}\right\|_{1, \Omega} \\
& +2 C_{2}\left\|\mathcal{V}^{*} \boldsymbol{\varphi}\right\|_{1, \Omega}\|\mathcal{V} \boldsymbol{\varphi}-\mathcal{V} \psi\|_{1, \Omega}\left\|\mathcal{V}^{*} \boldsymbol{\varphi}-\mathcal{V}^{*} \boldsymbol{\psi}\right\|_{1, \Omega} \\
& +C_{2}\left\|\mathcal{V}^{*} \boldsymbol{\varphi}-\mathcal{V}^{*} \boldsymbol{\psi}\right\|_{1, \Omega}^{2}\|\mathcal{V} \boldsymbol{\psi}\|_{1, \Omega}
\end{aligned}
$$

Owing to Lemmas 4.1, 4.4, 4.5 we have

$$
\begin{aligned}
\frac{\nu}{C_{0}^{2}}\left\|\mathcal{V}^{*} \boldsymbol{\varphi}-\mathcal{V}^{*} \boldsymbol{\psi}\right\|_{1, \Omega}^{2} \leq & C_{2}\left\|\mathcal{V}^{*} \boldsymbol{\varphi}-\mathcal{V}^{*} \boldsymbol{\psi}\right\|_{1, \Omega}^{2} C_{\alpha}^{*}\|\mathbf{f}\|_{-1, \Omega} \\
& +2 C_{2} C_{\alpha}^{*}\|\mathbf{f}\|_{-1, \Omega} C_{\gamma}\|\boldsymbol{\varphi}-\boldsymbol{\psi}\|_{1, \Omega}\left\|\mathcal{V}^{*} \boldsymbol{\varphi}-\mathcal{V}^{*} \boldsymbol{\psi}\right\|_{1, \Omega} \\
& +C_{2} C_{\alpha}\left\|\mathcal{V}^{*} \boldsymbol{\varphi}-\mathcal{V}^{*} \boldsymbol{\psi}\right\|_{1, \Omega}^{2}\|\boldsymbol{\psi}\|_{\Lambda}
\end{aligned}
$$

The inequality (38) now follows owing to (23), with

$$
C_{\gamma}^{*}=\frac{24 C_{0}^{4} C_{1} C_{2}\|\mathbf{f}\|_{-1, \Omega}}{\nu^{2}-4 C_{0}^{4} C_{2}\|\mathbf{f}\|_{-1, \Omega}}
$$

We define the Oseen extension to $\Omega$ of $\boldsymbol{\psi} \in \widetilde{\boldsymbol{\Lambda}}$ as being the solution $(\mathcal{O} \boldsymbol{\psi}, P \boldsymbol{\psi}) \in\left[H^{1}(\Omega)\right]^{d} \times L_{0}^{2}(\Omega)$ of:

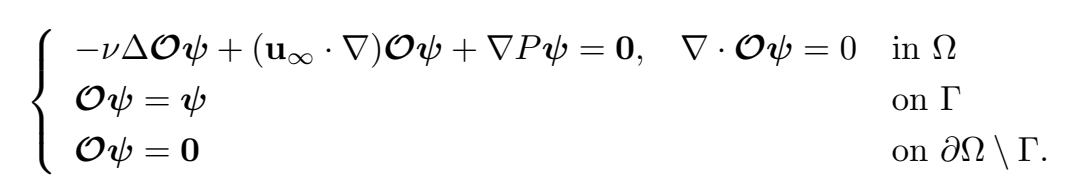

Lemma 4.7. For any $\boldsymbol{\psi} \in \widetilde{\boldsymbol{\Lambda}}$, the following estimate holds

$$
\|\mathcal{O} \psi\|_{1, \Omega} \leq C_{\beta}\|\psi\|_{\Lambda}
$$

where $C_{\beta}$ is a suitable positive constant depending on $\Omega, \nu,\left\|\mathbf{u}_{\infty}\right\|_{L^{\infty}(\Omega)}$ and $d$.

Proof. We follow the same guidelines of proof of Lemma 4.1. We subtract (25) from (40), we set $\mathbf{z}:=\boldsymbol{O} \boldsymbol{\psi}-\boldsymbol{U} \boldsymbol{\psi}$, $r:=P \boldsymbol{\psi}-R \boldsymbol{\psi}$ and we write the weak form of the resulting system with $\mathbf{v}=\mathbf{z}$ as test function, we have

$$
\nu a(\mathbf{z}, \mathbf{z})+e\left(\mathbf{u}_{\infty} ; \boldsymbol{U} \boldsymbol{\psi}, \mathbf{z}\right)=0 .
$$

By the Hölder inequality and the Sobolev embedding theorem there exists $C_{3}=C_{3}(d, \Omega)$ such that

$$
\left|e\left(\mathbf{u}_{\infty} ; \boldsymbol{U} \boldsymbol{\psi}, \mathbf{z}\right)\right| \leq C_{3}\left\|\mathbf{u}_{\infty}\right\|_{L^{\infty}(\Omega)}\|\boldsymbol{U} \boldsymbol{\psi}\|_{1, \Omega}\|\mathbf{z}\|_{1, \Omega}
$$

and by (26) we have

$$
\|\mathbf{z}\|_{1, \Omega} \leq \frac{C_{0}^{2} C_{1} C_{3}}{\nu}\left\|\mathbf{u}_{\infty}\right\|_{L^{\infty}(\Omega)}\|\boldsymbol{\psi}\|_{\Lambda} .
$$

The thesis follows with $C_{\beta}=C_{1}\left(1+C_{0}^{2} C_{3} \frac{\left\|\mathbf{u}_{\infty}\right\|_{L^{\infty}(\Omega)}}{\nu}\right)$. 
Finally we represent the solution $(\mathbf{u}, p)$ of the Oseen problem in $\Omega$ with forcing term $\mathbf{f}$ and Dirichlet boundary data $\psi$ on $\Gamma$ as being the sum of two terms:

$$
\mathbf{u}=\mathcal{O} \psi+\mathcal{O}^{*}, \quad p=P \boldsymbol{\psi}+P^{*}
$$

where the couple $\left(\mathcal{O}^{*}, P^{*}\right) \in\left[H_{0}^{1}(\Omega)\right]^{d} \times L_{0}^{2}(\Omega)$ is the solution of the following Oseen problem in $\Omega$ with homogeneous Dirichlet boundary data on $\partial \Omega$ :

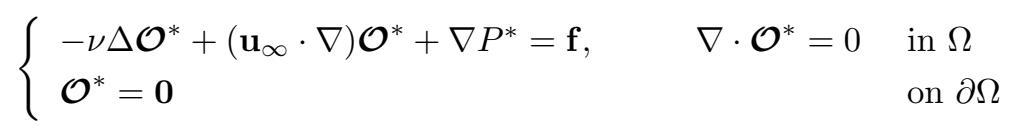

The representation (42) will be used in Theorem 5.2.

Remark 4.8. Owing to (15), the results proved in this section are still valid if, in the weak formulation associated to the Navier-Stokes equations, we take into account the skew-symmetric form of the convective term (see (13)).

\section{Steklov-Poincaré operators And the CONVERGEnCE TheOry}

For any $\boldsymbol{\psi} \in \widehat{\boldsymbol{\Lambda}}$, we denote by $\left(\mathcal{V}_{1} \boldsymbol{\psi}, \Pi_{1} \boldsymbol{\psi}\right) \in\left[V_{1}\right]^{d} \times L_{0}^{2}\left(\Omega_{1}\right)$ the Navier-Stokes extension of $\boldsymbol{\psi} \in \widehat{\boldsymbol{\Lambda}}$ to $\Omega_{1}$

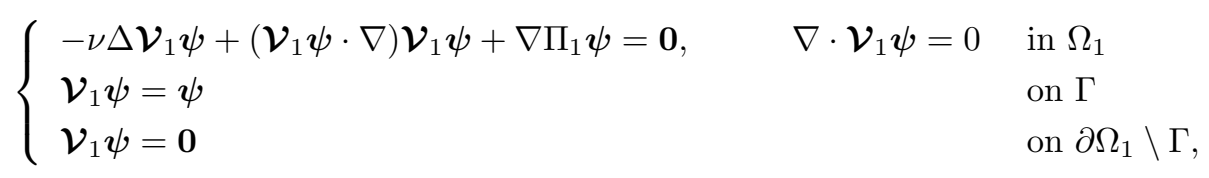

whereas $\left(\mathcal{O}_{2} \boldsymbol{\psi}, P_{2} \boldsymbol{\psi}\right) \in\left[V_{2}\right]^{d} \times L_{0}^{2}\left(\Omega_{2}\right)$ is the Oseen extension of $\boldsymbol{\psi} \in \widehat{\boldsymbol{\Lambda}}$ to $\Omega_{2}$,

$$
\left\{\begin{array}{lll}
-\nu \Delta \mathcal{O}_{2} \boldsymbol{\psi}+\left(\mathbf{u}_{\infty} \cdot \nabla\right) \mathcal{O}_{2} \boldsymbol{\psi}+\nabla P_{2} \boldsymbol{\psi}=\mathbf{0}, & \nabla \cdot \mathcal{O}_{2} \boldsymbol{\psi}=0 & \text { in } \Omega_{2} \\
\mathcal{O}_{2} \boldsymbol{\psi}=\boldsymbol{\psi} & \text { on } \Gamma \\
\mathcal{O}_{2} \boldsymbol{\psi}=\mathbf{0} & \text { on } \partial \Omega_{2} \backslash \Gamma .
\end{array}\right.
$$

We formally define the local Steklov-Poincaré operators $S_{i}, i=1,2$, on the trace space $\widehat{\boldsymbol{\Lambda}}$ into its dual $\widehat{\boldsymbol{\Lambda}}^{\prime}$ as follows:

$$
\begin{aligned}
& S_{1}(\boldsymbol{\psi}):=\nu \frac{\partial \mathcal{V}_{1} \boldsymbol{\psi}}{\partial \mathbf{n}}-\Pi_{1} \boldsymbol{\psi} \mathbf{n}-\frac{1}{2}\left(\mathcal{V}_{1} \boldsymbol{\psi} \cdot \mathbf{n}\right) \mathcal{V}_{1} \boldsymbol{\psi} \\
& S_{2} \boldsymbol{} \boldsymbol{:}=-\nu \frac{\partial \mathcal{O}_{2} \boldsymbol{\psi}}{\partial \mathbf{n}}+P_{2} \boldsymbol{\psi} \mathbf{n}+\frac{1}{2}\left(\mathbf{u}_{\infty} \cdot \mathbf{n}\right) \mathcal{O}_{2} \boldsymbol{\psi}
\end{aligned}
$$

Now we can define the Steklov-Poincaré operator $S$ as

$$
S(\boldsymbol{\psi}):=S_{1}(\boldsymbol{\psi})+S_{2} \psi \quad \forall \boldsymbol{\psi} \in \widehat{\Lambda} .
$$

Lemma 5.1. We have

$$
\left\langle S_{1}(\boldsymbol{\psi}), \boldsymbol{\mu}\right\rangle=d_{1}\left(\mathcal{V}_{1} \boldsymbol{\psi} ; \mathcal{V}_{1} \boldsymbol{\psi}, \boldsymbol{\mathcal { R }}_{1} \boldsymbol{\mu}\right)+b_{1}\left(\boldsymbol{\mathcal { R }}_{1} \boldsymbol{\mu}, \Pi_{1} \boldsymbol{\psi}\right) \quad \forall \boldsymbol{\psi}, \boldsymbol{\mu} \in \widehat{\boldsymbol{\Lambda}}
$$

and

$$
\left\langle S_{2} \boldsymbol{\psi}, \boldsymbol{\mu}\right\rangle=d_{2}\left(\mathbf{u}_{\infty} ; \mathcal{O}_{2} \boldsymbol{\psi}, \boldsymbol{\mathcal { R }}_{2} \boldsymbol{\mu}\right)+b_{2}\left(\boldsymbol{\mathcal { R }}_{2} \boldsymbol{\mu}, P_{2} \boldsymbol{\psi}\right) \quad \forall \boldsymbol{\psi}, \boldsymbol{\mu} \in \widehat{\boldsymbol{\Lambda}}
$$

These characterizations hold for any possible choice of the extension operator $\mathcal{R}_{i} \boldsymbol{\mu}, i=1,2$. 
Proof. Applying Green's formula, we have:

$$
\begin{aligned}
\left\langle S_{1}(\boldsymbol{\psi}), \boldsymbol{\mu}\right\rangle= & \left\langle\operatorname{div}\left(\nu \nabla \mathcal{V}_{1} \boldsymbol{\psi}-\Pi_{1} \psi \mathbf{I}-\frac{1}{2} \mathcal{V}_{1} \boldsymbol{\psi} \mathcal{V}_{1} \boldsymbol{\psi}\right), \boldsymbol{\mathcal { R }}_{1} \boldsymbol{\mu}\right\rangle \\
& +\left\langle\nu \nabla \mathcal{V}_{1} \boldsymbol{\psi}-\Pi_{1} \psi \mathbf{I}-\frac{1}{2} \mathcal{V}_{1} \boldsymbol{\psi} \mathcal{V}_{1} \boldsymbol{\psi}, \nabla \boldsymbol{\mathcal { R }}_{1} \boldsymbol{\mu}\right\rangle
\end{aligned}
$$

where I is the identity tensor. From (44) we deduce

$$
\begin{aligned}
\left\langle S_{1}(\boldsymbol{\psi}), \boldsymbol{\mu}\right\rangle= & \frac{1}{2}\left(\left(\mathcal{V}_{1} \boldsymbol{\psi} \cdot \nabla\right) \mathcal{V}_{1} \boldsymbol{\psi}, \boldsymbol{\mathcal { R }}_{1} \boldsymbol{\mu}\right)_{\Omega_{1}}+\left(\nu \nabla \mathcal{V}_{1} \boldsymbol{\psi}, \nabla \boldsymbol{\mathcal { R }}_{1} \boldsymbol{\mu}\right)_{\Omega_{1}} \\
& -\frac{1}{2}\left(\left(\mathcal{V}_{1} \psi \cdot \nabla\right) \boldsymbol{\mathcal { R }}_{1} \boldsymbol{\mu}, \nabla \mathcal{V}_{1} \psi\right)_{\Omega_{1}}-\left(\nabla \cdot \boldsymbol{\mathcal { R }}_{1} \boldsymbol{\mu}, \Pi_{1} \boldsymbol{\psi}\right)
\end{aligned}
$$

then the result (45) follows. With similar arguments we can prove (46).

Let us define the operator $\chi: \widehat{\Lambda} \longrightarrow \widehat{\boldsymbol{\Lambda}}^{\prime}$ as

$$
\begin{aligned}
& \langle\chi(\boldsymbol{\psi}), \boldsymbol{\mu}\rangle:=\mathcal{F}_{1}\left(\mathcal{R}_{1} \boldsymbol{\mu}\right)-d_{1}\left(\mathcal{V}_{1}^{*} \boldsymbol{\psi} ; \mathcal{V}_{1}^{*} \boldsymbol{\psi}, \mathcal{R}_{1} \boldsymbol{\mu}\right)-b_{1}\left(\mathcal{R}_{1} \boldsymbol{\mu}, \Pi_{1}^{*} \boldsymbol{\psi}\right) \\
& -\frac{1}{2} e_{1}\left(\mathcal{V}_{1}^{*} \boldsymbol{\psi} ; \mathcal{V}_{1} \boldsymbol{\psi}, \boldsymbol{\mathcal { R }}_{1} \boldsymbol{\mu}\right)+\frac{1}{2} e_{1}\left(\mathcal{V}_{1}^{*} \boldsymbol{\psi} ; \mathcal{R}_{1} \boldsymbol{\mu}, \mathcal{V}_{1} \boldsymbol{\psi}\right) \\
& -\frac{1}{2} e_{1}\left(\mathcal{V}_{1} \boldsymbol{\psi} ; \mathcal{V}_{1}^{*} \boldsymbol{\psi}, \boldsymbol{\mathcal { R }}_{1} \boldsymbol{\mu}\right)+\frac{1}{2} e_{1}\left(\mathcal{V}_{1} \boldsymbol{\psi} ; \boldsymbol{\mathcal { R }}_{1} \boldsymbol{\mu}, \mathcal{V}_{1}^{*} \boldsymbol{\psi}\right) \\
& +\mathcal{F}_{2}\left(\boldsymbol{\mathcal { R }}_{2} \boldsymbol{\mu}\right)-d_{2}\left(\mathbf{u}_{\infty} ; \mathcal{O}_{2}^{*}, \boldsymbol{\mathcal { R }}_{2} \boldsymbol{\mu}\right)-b_{2}\left(\boldsymbol{\mathcal { R }}_{2} \boldsymbol{\mu}, P_{2}^{*}\right) \quad \forall \boldsymbol{\mu} \in \widehat{\boldsymbol{\Lambda}}
\end{aligned}
$$

Note that $\chi(\psi) \equiv \mathbf{0}$ when $\mathbf{f} \equiv \mathbf{0}$.

The following theorem states the equivalence between the two-domain heterogeneous problem (16) and the formulation on the interface.

Theorem 5.2. If $\left(\mathbf{u}_{1}, p_{1}\right)$ and $\left(\mathbf{u}_{2}, p_{2}\right)$ are the solutions of the multidomain problem (16), then the function $\boldsymbol{\lambda}=\mathbf{u}_{1 \mid \Gamma}=\mathbf{u}_{2 \mid \Gamma}$, satisfies the following Steklov-Poincaré equation on $\Gamma$

$$
\text { find } \boldsymbol{\lambda} \in \widehat{\boldsymbol{\Lambda}}: \quad\langle S(\boldsymbol{\lambda}), \boldsymbol{\mu}\rangle=\langle\chi(\boldsymbol{\lambda}), \boldsymbol{\mu}\rangle \quad \forall \boldsymbol{\mu} \in \widehat{\boldsymbol{\Lambda}} .
$$

Conversely, should a solution $\boldsymbol{\lambda}$ of (48) be available, we could recover the solution of (16) by setting

$$
\begin{array}{ll}
\mathbf{u}_{1}=\mathcal{V}_{1} \boldsymbol{\lambda}+\mathcal{V}_{1}^{*} \boldsymbol{\lambda} & p_{1}=\Pi_{1} \boldsymbol{\lambda}+\Pi_{1}^{*} \boldsymbol{\lambda}+\hat{p}_{1} \\
\mathbf{u}_{2}=\mathcal{O}_{2} \boldsymbol{\lambda}+\mathcal{O}_{2}^{*} & p_{2}=P_{2} \boldsymbol{\lambda}+P_{2}^{*}+\hat{p}_{2},
\end{array}
$$

where the two constants $\hat{p}_{1}$ and $\hat{p}_{2}$ are obtained by solving the $2 \times 2$ linear system

$$
\left\{\begin{array}{l}
\hat{p}_{1}-\hat{p}_{2}=\frac{1}{\operatorname{meas}(\Gamma)}\langle S(\boldsymbol{\lambda})-\boldsymbol{\chi}(\boldsymbol{\lambda}), \mathbf{n}\rangle \\
\hat{p}_{1} \text { meas }\left(\Omega_{1}\right)+\hat{p}_{2} \operatorname{meas}\left(\Omega_{2}\right)=0 .
\end{array}\right.
$$

Proof. Let $\left(\mathbf{u}_{i}, p_{i}\right) \in\left[V_{i}\right]^{d} \times L^{2}\left(\Omega_{i}\right), i=1,2$ be the solutions of the two-domain problem (16). They can be written has

$$
\begin{array}{ll}
\mathbf{u}_{1}=\mathcal{V}_{1} \mathbf{u}_{1 \mid \Gamma}+\mathcal{V}_{1}^{*} \mathbf{u}_{1 \mid \Gamma}, & p_{1}=\Pi_{1} \mathbf{u}_{1 \mid \Gamma}+\Pi_{1}^{*} \mathbf{u}_{1 \mid \Gamma}+\bar{p}_{1}, \\
\mathbf{u}_{2}=\mathcal{O}_{2} \mathbf{u}_{2 \mid \Gamma}+\mathcal{O}_{2}^{*}, & p_{2}=P_{2} \mathbf{u}_{2 \mid \Gamma}+P_{2}^{*}+\bar{p}_{2},
\end{array}
$$


where the constants

$$
\bar{p}_{i}:=\frac{1}{\operatorname{meas}\left(\Omega_{i}\right)} \int_{\Omega_{i}} p_{i} \mathrm{~d} \Omega_{i}, \quad i=1,2
$$

are added to restore the correct mean value of the pressure in the domain $\Omega_{1}$ and in the whole domain $\Omega$. We consider the second interface condition in (16) where we express $\left(\mathbf{u}_{i}, p_{i}\right)$ as in (51). Then

$$
\begin{aligned}
& d_{2}\left(\mathbf{u}_{\infty} ; \mathcal{O}_{2} \mathbf{u}_{2 \mid \Gamma}, \mathcal{R}_{2} \boldsymbol{\mu}\right)+b_{2}\left(\mathcal{R}_{2} \boldsymbol{\mu}, P_{2} \mathbf{u}_{2 \mid \Gamma}\right) \\
& +d_{2}\left(\mathbf{u}_{\infty} ; \mathcal{O}_{2}^{*}, \mathcal{R}_{2} \boldsymbol{\mu}\right)+b_{2}\left(\mathcal{R}_{2} \boldsymbol{\mu}, P_{2}^{*}\right)+\bar{p}_{2} b_{2}\left(\mathcal{R}_{2} \boldsymbol{\mu}, 1\right) \\
& +d_{1}\left(\mathcal{V}_{1} \mathbf{u}_{1 \mid \Gamma} ; \mathcal{V}_{1} \mathbf{u}_{1 \mid \Gamma}, \mathcal{R}_{1} \boldsymbol{\mu}\right)+b_{1}\left(\mathcal{R}_{1} \boldsymbol{\mu}, \Pi_{1} \mathbf{u}_{1 \mid \Gamma}\right) \\
& +d_{1}\left(\mathcal{V}_{1}^{*} \mathbf{u}_{1 \mid \Gamma} ; \mathcal{V}_{1}^{*} \mathbf{u}_{1 \mid \Gamma}, \mathcal{R}_{1} \boldsymbol{\mu}\right)+b_{1}\left(\mathcal{R}_{1} \boldsymbol{\mu}, \Pi_{1}^{*} \mathbf{u}_{1 \mid \Gamma}\right)+\bar{p}_{1} b_{1}\left(\mathcal{R}_{1} \boldsymbol{\mu}, 1\right) \\
& +\frac{1}{2} e_{1}\left(\mathcal{V}_{1}^{*} \mathbf{u}_{1 \mid \Gamma} ; \mathcal{V}_{1} \mathbf{u}_{1 \mid \Gamma}, \mathcal{R}_{1} \boldsymbol{\mu}\right)-\frac{1}{2} e_{1}\left(\mathcal{V}_{1}^{*} \mathbf{u}_{1 \mid \Gamma} ; \mathcal{R}_{1} \boldsymbol{\mu}, \mathcal{V}_{1} \mathbf{u}_{1 \mid \Gamma}\right) \\
& +\frac{1}{2} e_{1}\left(\mathcal{V}_{1} \mathbf{u}_{1 \mid \Gamma} ; \mathcal{V}_{1}^{*} \mathbf{u}_{1 \mid \Gamma}, \mathcal{R}_{1} \boldsymbol{\mu}\right)-\frac{1}{2} e_{1}\left(\mathcal{V}_{1} \mathbf{u}_{1 \mid \Gamma} ; \mathcal{R}_{1} \boldsymbol{\mu}, \mathcal{V}_{1}^{*} \mathbf{u}_{1 \mid \Gamma}\right) \\
& =\mathcal{F}_{1}\left(\mathcal{R}_{1} \boldsymbol{\mu}\right)+\mathcal{F}_{2}\left(\mathcal{R}_{2} \boldsymbol{\mu}\right) \quad \forall \boldsymbol{\mu} \in[\Lambda]^{d}
\end{aligned}
$$

By (45), (46) and the definition of $\chi(47)$ the previous relation can be equivalent rewritten as

$$
\left\langle S\left(\mathbf{u}_{\mid \Gamma}\right), \boldsymbol{\mu}\right\rangle=\left\langle\chi\left(\mathbf{u}_{\mid \Gamma}\right), \boldsymbol{\mu}\right\rangle-\sum_{i=1}^{2} \bar{p}_{i} b_{i}\left(\boldsymbol{\mathcal { R }}_{i} \boldsymbol{\mu}, 1\right)
$$

We observe that

$$
-\sum_{i=1}^{2} \bar{p}_{i} b_{i}\left(\boldsymbol{\mathcal { R }}_{i} \boldsymbol{\mu}, 1\right)=\left(\bar{p}_{1}-\bar{p}_{2}\right) \int_{\Gamma} \boldsymbol{\mu} \cdot \mathbf{n} \mathrm{d} \gamma
$$

we therefore obtain (48) owing to the definition (17) of $\widehat{\Lambda}$.

Conversely, let $\boldsymbol{\lambda}$ be the solution of (48); we are going to show that the functions

$$
\begin{array}{ll}
\mathbf{u}_{1}=\mathcal{V}_{1} \boldsymbol{\lambda}+\mathcal{V}_{1}^{*} \boldsymbol{\lambda}, & p_{1}=\Pi_{1} \boldsymbol{\lambda}+\Pi_{1}^{*} \boldsymbol{\lambda}+\hat{p}_{1} \\
\mathbf{u}_{2}=\mathcal{O}_{2} \boldsymbol{\lambda}+\mathcal{O}_{2}^{*}, & p_{2}=P_{2} \mathbf{u}_{2 \mid \Gamma}+P_{2}^{*}+\hat{p}_{2}
\end{array}
$$

satisfy the coupled problem (16). Since $\left(\mathcal{V}_{1} \boldsymbol{\lambda}, \Pi_{1} \boldsymbol{\lambda}\right),\left(\mathcal{V}_{1}^{*} \boldsymbol{\lambda}, \Pi_{1}^{*} \boldsymbol{\lambda}\right),\left(\mathcal{O}_{2} \boldsymbol{\lambda}, P_{2} \boldsymbol{\lambda}\right)$, and $\left(\mathcal{O}_{2}^{*}, P_{2}^{*}\right)$ satisfy (21), (36), (40) and (43), respectively, it is straightforward to prove that the first four equations of (16) are verified.

To prove the continuity equation in $\Omega_{2}$ we take any function $q_{2} \in L^{2}\left(\Omega_{2}\right)$ and define

$$
\bar{q}_{2}:=\frac{1}{\operatorname{meas}\left(\Omega_{2}\right)} \int_{\Omega_{2}} q_{2} \mathrm{~d} \Omega_{2},
$$

so that $q_{2}-\bar{q}_{2} \in L_{0}^{2}\left(\Omega_{2}\right)$. We have

$$
b_{2}\left(\mathbf{u}_{2}, q_{2}\right)=b_{2}\left(\mathbf{u}_{2}, q_{2}-\bar{q}_{2}\right)+b_{2}\left(\mathbf{u}_{2}, \bar{q}_{2}\right)=\bar{q}_{2} b_{2}\left(\mathbf{u}_{2}, 1\right)=\bar{q}_{2} \int_{\Gamma} \boldsymbol{\lambda} \cdot \mathbf{n} \mathrm{d} \gamma=0 .
$$


Now using (52) we have:

$$
\begin{aligned}
& d_{1}\left(\mathbf{u}_{1} ; \mathbf{u}_{1}, \boldsymbol{\mathcal { R }}_{1} \boldsymbol{\mu}\right)+b_{1}\left(\mathcal{R}_{1} \boldsymbol{\mu}, p_{1}\right)+d_{2}\left(\mathbf{u}_{\infty} ; \mathbf{u}_{2}, \boldsymbol{\mathcal { R }}_{2} \boldsymbol{\mu}\right)+b_{2}\left(\mathcal{R}_{2} \boldsymbol{\mu}, p_{2}\right) \\
& =d_{1}\left(\mathcal{V}_{1} \boldsymbol{\lambda} ; \mathcal{V}_{1} \boldsymbol{\lambda}, \boldsymbol{\mathcal { R }}_{1} \boldsymbol{\mu}\right)+b_{1}\left(\boldsymbol{\mathcal { R }}_{1} \boldsymbol{\mu}, \Pi_{1} \boldsymbol{\mu}\right)+d_{2}\left(\mathbf{u}_{\infty} ; \mathcal{O}_{2} \boldsymbol{\lambda}, \mathcal{R}_{2} \boldsymbol{\mu}\right)+b_{2}\left(\mathcal{R}_{2} \boldsymbol{\mu}, P_{2} \boldsymbol{\lambda}\right) \\
& +d_{1}\left(\mathcal{V}_{1}^{*} \boldsymbol{\lambda} ; \mathcal{V}_{1}^{*} \boldsymbol{\lambda}, \boldsymbol{\mathcal { R }}_{1} \boldsymbol{\mu}\right)+b_{1}\left(\mathcal{R}_{1} \boldsymbol{\mu}, \Pi_{1}^{*} \boldsymbol{\mu}\right)+d_{2}\left(\mathbf{u}_{\infty} ; \mathcal{O}_{2}^{*}, \mathcal{R}_{2} \boldsymbol{\mu}\right)+b_{2}\left(\mathcal{R}_{2} \boldsymbol{\mu}, P_{2}^{*}\right) \\
& \quad+\frac{1}{2} e_{1}\left(\mathcal{V}_{1} \boldsymbol{\lambda} ; \mathcal{V}_{1}^{*} \boldsymbol{\lambda}, \boldsymbol{\mathcal { R }}_{1} \boldsymbol{\mu}\right)-\frac{1}{2} e_{1}\left(\mathcal{V}_{1} \boldsymbol{\lambda} ; \boldsymbol{\mathcal { R }}_{1} \boldsymbol{\mu}, \mathcal{V}_{1}^{*} \boldsymbol{\lambda}\right) \\
& \quad+\frac{1}{2} e_{1}\left(\mathcal{V}_{1}^{*} \boldsymbol{\lambda} ; \mathcal{V}_{1} \boldsymbol{\lambda}, \mathcal{R}_{1} \boldsymbol{\mu}\right)+\frac{1}{2} e_{1}\left(\mathcal{V}_{1}^{*} \boldsymbol{\lambda} ; \mathcal{R}_{1} \boldsymbol{\mu}, \mathcal{V}_{1} \boldsymbol{\lambda}\right) \\
& \quad+b_{1}\left(\boldsymbol{\mathcal { R }}_{1} \boldsymbol{\mu}, \hat{p}_{1}\right)+b_{2}\left(\boldsymbol{\mathcal { R }}_{2} \boldsymbol{\mu}, \hat{p}_{2}\right) \\
& =\left\langle S_{1}(\boldsymbol{\lambda}), \boldsymbol{\mu}\right\rangle+\left\langle S_{2} \boldsymbol{\lambda}, \boldsymbol{\mu}\right\rangle-\langle\boldsymbol{\chi}(\boldsymbol{\lambda}), \boldsymbol{\mu}\rangle+\sum_{i=1}^{2} \mathcal{F}_{i}\left(\boldsymbol{\mathcal { R }}_{i} \boldsymbol{\mu}\right)-\left(\hat{p}_{1}-\hat{p}_{2}\right) \int_{\Gamma} \boldsymbol{\mu} \cdot \mathbf{n} \mathrm{d} \gamma
\end{aligned}
$$

The second transmission condition in (16) is therefore satisfied, provided that

$$
\langle S(\boldsymbol{\lambda})-\boldsymbol{\chi}(\boldsymbol{\lambda}), \boldsymbol{\mu}\rangle-\left(\hat{p}_{1}-\hat{p}_{2}\right) \int_{\Gamma} \boldsymbol{\mu} \cdot \mathbf{n} \mathrm{d} \gamma=0
$$

We define

$$
\bar{\mu}_{\Gamma}:=\frac{1}{\operatorname{meas}(\Gamma)} \int_{\Gamma} \boldsymbol{\mu} \cdot \mathbf{n} \mathrm{d} \gamma
$$

and we note that $\boldsymbol{\mu}-\bar{\mu}_{\Gamma} \mathbf{n} \in \widehat{\boldsymbol{\Lambda}}\left(\mathbf{n} \in[\Lambda]^{d}\right.$ since $\Gamma$ is a subset of a Lipschitz boundary $\left.\partial \Omega\right)$. It follows that

$$
\begin{aligned}
\langle S(\boldsymbol{\lambda})-\chi(\boldsymbol{\lambda}), \boldsymbol{\mu}\rangle & =\left\langle S(\boldsymbol{\lambda})-\chi(\boldsymbol{\lambda}), \boldsymbol{\mu}-\bar{\mu}_{\Gamma} \mathbf{n}\right\rangle+\left\langle S(\boldsymbol{\lambda})-\chi(\boldsymbol{\lambda}), \bar{\mu}_{\Gamma} \mathbf{n}\right\rangle \\
& =\bar{\mu}_{\Gamma}\langle S(\boldsymbol{\lambda})-\chi(\boldsymbol{\lambda}), \mathbf{n}\rangle
\end{aligned}
$$

thus we obtain (53) owing to the first equation of (50).

Finally, the last equation in (16) is nothing but the second equation in (50).

In the special case where we take as extension operators $\boldsymbol{R}_{1} \boldsymbol{\mu}=\mathcal{V}_{1} \boldsymbol{\mu}$ and $\boldsymbol{R}_{2} \boldsymbol{\mu}=\mathcal{O}_{2} \boldsymbol{\mu}$, we obtain

$$
\left\langle S_{1}(\boldsymbol{\psi}), \boldsymbol{\mu}\right\rangle=d_{1}\left(\mathcal{V}_{1} \boldsymbol{\psi} ; \mathcal{V}_{1} \boldsymbol{\psi}, \mathcal{V}_{1} \boldsymbol{\mu}\right), \quad\left\langle S_{2} \boldsymbol{\psi}, \boldsymbol{\mu}\right\rangle=d_{2}\left(\mathbf{u}_{\infty} ; \mathcal{O}_{2} \boldsymbol{\psi}, \mathcal{O}_{2} \boldsymbol{\mu}\right)
$$

using the definitions (45), (46) and the property that both $\mathcal{V}_{1} \boldsymbol{\mu}$ and $\mathcal{O}_{2} \boldsymbol{\mu}$ are divergence-free.

Moreover,

$$
\begin{aligned}
& \langle\chi(\psi), \boldsymbol{\mu}\rangle=\mathcal{F}_{1}\left(\boldsymbol{R}_{1} \boldsymbol{\mu}\right)-d_{1}\left(\mathcal{V}_{1}^{*} \boldsymbol{\psi} ; \mathcal{V}_{1}^{*} \boldsymbol{\psi}, \boldsymbol{\mathcal { R }}_{1} \boldsymbol{\mu}\right) \\
& -\frac{1}{2} e_{1}\left(\mathcal{V}_{1}^{*} \boldsymbol{\psi} ; \mathcal{V}_{1} \boldsymbol{\psi}, \boldsymbol{\mathcal { R }}_{1} \boldsymbol{\mu}\right)+\frac{1}{2} e_{1}\left(\mathcal{V}_{1}^{*} \boldsymbol{\psi} ; \boldsymbol{R}_{1} \boldsymbol{\mu}, \mathcal{V}_{1} \boldsymbol{\psi}\right) \\
& -\frac{1}{2} e_{1}\left(\mathcal{V}_{1} \boldsymbol{\psi} ; \mathcal{V}_{1}^{*} \boldsymbol{\psi}, \boldsymbol{\mathcal { R }}_{1} \boldsymbol{\mu}\right)+\frac{1}{2} e_{1}\left(\mathcal{V}_{1} \boldsymbol{\psi} ; \boldsymbol{\mathcal { R }}_{1} \boldsymbol{\mu}, \mathcal{V}_{1}^{*} \boldsymbol{\psi}\right) \\
& +\mathcal{F}_{2}\left(\boldsymbol{\mathcal { R }}_{2} \boldsymbol{\mu}\right)-d_{2}\left(\mathbf{u}_{\infty} ; \mathcal{O}_{2}^{*}, \boldsymbol{\mathcal { R }}_{2} \boldsymbol{\mu}\right)
\end{aligned}
$$

In this way it can be proven that the non-linear operator $S_{1}$ is bounded and non-negative, while $S_{2}$, which is linear, is coercive and continuous, as stated by the following lemmas. 
Lemma 5.3. There exist two positive constants $\alpha_{1}=\alpha_{1}\left(\nu, \Omega_{2}\right)$ and $\alpha_{2}=\alpha_{2}\left(\nu, \Omega_{2},\left\|\mathbf{u}_{\infty}\right\|_{L^{\infty}\left(\Omega_{2}\right)}, d\right)$ such that

$$
\left\langle S_{2} \boldsymbol{\psi}, \boldsymbol{\psi} \geq \alpha_{1}\|\boldsymbol{\psi}\|_{\Lambda}^{2} \quad \forall \boldsymbol{\psi} \in \widehat{\Lambda}\right.
$$

and

$$
\left|\left\langle S_{2} \boldsymbol{\psi}, \boldsymbol{\mu}\right\rangle\right| \leq \alpha_{2}\|\boldsymbol{\psi}\|_{\Lambda}\|\boldsymbol{\mu}\|_{\Lambda} \quad \forall \boldsymbol{\psi}, \boldsymbol{\mu} \in \widehat{\boldsymbol{\Lambda}}
$$

Proof. By (55) and (26) we have

$$
\left\langle S_{2} \boldsymbol{\psi}, \boldsymbol{\psi}\right\rangle \frac{\nu}{C_{0}^{2}}\left\|\mathcal{O}_{2} \boldsymbol{\psi}\right\|_{1, \Omega_{2}}^{2}
$$

Owing to the continuity of the trace operator, $\exists C_{4}=C_{4}\left(\Omega_{2}\right)>0$ such that

$$
\|\boldsymbol{\psi}\|_{\Lambda} \leq C_{4}\left\|\mathcal{O}_{2} \psi\right\|_{1, \Omega_{2}}
$$

then (57) follows with $\alpha_{1}=\frac{\nu}{C_{0}^{2} C_{4}^{2}}$.

By (55), (41) and Lemma 4.7 we have

$$
\begin{aligned}
\left|\left\langle S_{2} \boldsymbol{\psi}, \boldsymbol{\mu}\right\rangle\right| & =\left|d_{2}\left(\mathbf{u}_{\infty}, \mathcal{O}_{2} \boldsymbol{\psi}, \mathcal{O}_{2} \boldsymbol{\mu}\right)\right| \\
& \leq\left(\nu+C_{3}\left\|\mathbf{u}_{\infty}\right\|_{L^{\infty}\left(\Omega_{2}\right)}\right)\left\|\mathcal{O}_{2} \boldsymbol{\psi}\right\|_{1, \Omega_{2}}\left\|\mathcal{O}_{2} \boldsymbol{\mu}\right\|_{1, \Omega_{2}} \\
& \leq\left(\nu+C_{3}\left\|\mathbf{u}_{\infty}\right\|_{L^{\infty}\left(\Omega_{2}\right)}\right) C_{\beta}^{2}\|\boldsymbol{\psi}\|_{\Lambda}\|\boldsymbol{\mu}\|_{\Lambda},
\end{aligned}
$$

whence (58) follows with $\alpha_{2}=\left(\nu+C_{3}\left\|\mathbf{u}_{\infty}\right\|_{L^{\infty}\left(\Omega_{2}\right)}\right) C_{\beta}^{2}$.

We define the ball

$$
\widehat{\boldsymbol{\Lambda}}_{\nu}:=\left\{\boldsymbol{\mu} \in \widehat{\boldsymbol{\Lambda}}:\|\boldsymbol{\mu}\|_{\Lambda} \leq \zeta_{\nu}\right\}
$$

where the constant $\zeta_{\nu}$ is introduced in (23).

Lemma 5.4. There exists a positive constant $\beta_{2}$, depending on $\nu, \Omega,\left\|\mathbf{u}_{\infty}\right\|_{L^{\infty}\left(\Omega_{2}\right)}$ and d, such that

$$
\left\langle S_{1}(\boldsymbol{\varphi})-S_{1}(\boldsymbol{\psi}), \boldsymbol{\mu}\right\rangle \leq \beta_{2}\|\boldsymbol{\varphi}-\boldsymbol{\psi}\|_{\Lambda}\|\boldsymbol{\mu}\|_{\Lambda}, \quad \forall \boldsymbol{\varphi}, \boldsymbol{\psi}, \boldsymbol{\mu} \in \widehat{\boldsymbol{\Lambda}}_{\nu}
$$

Proof. We use (55) and we add and subtract the terms $\frac{1}{2}\left[e_{1}\left(\mathcal{V}_{1} \boldsymbol{\varphi} ; \mathcal{V}_{1} \boldsymbol{\psi}, \mathcal{V}_{1} \boldsymbol{\mu}\right)+e_{1}\left(\mathcal{V}_{1} \boldsymbol{\varphi} ; \mathcal{V}_{1} \boldsymbol{\mu}, \mathcal{V}_{1} \boldsymbol{\psi}\right)\right]$. Then

$$
\begin{aligned}
& \left\langle S_{1}(\boldsymbol{\varphi})-S_{1}(\boldsymbol{\psi}), \boldsymbol{\mu}\right\rangle=d_{1}\left(\mathcal{V}_{1} \boldsymbol{\varphi} ; \mathcal{V}_{1} \boldsymbol{\varphi}, \mathcal{V}_{1} \boldsymbol{\mu}\right)-d_{1}\left(\mathcal{V}_{1} \boldsymbol{\psi} ; \mathcal{V}_{1} \boldsymbol{\psi}, \mathcal{V}_{1} \boldsymbol{\mu}\right) \\
& =\nu a_{1}\left(\mathcal{V}_{1} \boldsymbol{\varphi}-\mathcal{V}_{1} \boldsymbol{\psi}, \mathcal{V}_{1} \boldsymbol{\mu}\right)+\frac{1}{2} e_{1}\left(\mathcal{V}_{1} \boldsymbol{\varphi} ; \mathcal{V}_{1} \boldsymbol{\varphi}-\mathcal{V}_{1} \boldsymbol{\psi}, \mathcal{V}_{1} \boldsymbol{\mu}\right) \\
& -\frac{1}{2} e_{1}\left(\mathcal{V}_{1} \boldsymbol{\varphi}-\mathcal{V}_{1} \boldsymbol{\psi} ; \mathcal{V}_{1} \boldsymbol{\mu}, \mathcal{V}_{1} \boldsymbol{\psi}\right)+\frac{1}{2} e_{1}\left(\mathcal{V}_{1} \boldsymbol{\varphi}-\mathcal{V}_{1} \boldsymbol{\psi} ; \mathcal{V}_{1} \boldsymbol{\psi}, \mathcal{V}_{1} \boldsymbol{\mu}\right) \\
& -\frac{1}{2} e_{1}\left(\mathcal{V}_{1} \boldsymbol{\varphi} ; \mathcal{V}_{1} \boldsymbol{\mu}, \mathcal{V}_{1} \boldsymbol{\varphi}-\mathcal{V}_{1} \psi\right)
\end{aligned}
$$


Owing to the continuity of the forms $a_{1}(\cdot, \cdot)$ and $e_{1}(\cdot ; \cdot, \cdot)$ we obtain

$$
\begin{aligned}
\left|\left\langle S_{1}(\boldsymbol{\varphi})-S_{1}(\boldsymbol{\psi}), \boldsymbol{\mu}\right\rangle\right| \leq & \nu\left\|\mathcal{V}_{1} \boldsymbol{\varphi}-\mathcal{V}_{1} \boldsymbol{\psi}\right\|_{1, \Omega_{1}}\left\|\mathcal{V}_{1} \boldsymbol{\mu}\right\|_{1, \Omega_{1}} \\
& +C_{2}\left\|\mathcal{V}_{1} \boldsymbol{\varphi}-\mathcal{V}_{1} \boldsymbol{\psi}\right\|_{1, \Omega_{1}}\left\|\mathcal{V}_{1} \boldsymbol{\varphi}\right\|_{1, \Omega_{1}}\left\|\mathcal{V}_{1} \boldsymbol{\mu}\right\|_{1, \Omega_{1}} \\
& +C_{2}\left\|\mathcal{V}_{1} \boldsymbol{\varphi}-\mathcal{V}_{1} \boldsymbol{\psi}\right\|_{1, \Omega_{1}}\left\|\mathcal{V}_{1} \boldsymbol{\mu}\right\|_{1, \Omega_{1}}\left\|\mathcal{V}_{1} \boldsymbol{\psi}\right\|_{1, \Omega_{1}}
\end{aligned}
$$

Now (60) follows with $\beta_{2}=\frac{9}{2} \nu C_{1}^{2}\left(\frac{1}{C_{0}^{2}}+1\right)$ by applying Lemmas 4.1 and 4.4 and using the definition of $\widehat{\Lambda}_{\nu}$ $(59)$.

Lemma 5.5. We have

$$
\left\langle S_{1}(\boldsymbol{\varphi})-S_{1}(\boldsymbol{\psi}), \boldsymbol{\varphi}-\boldsymbol{\psi}\right\rangle \geq 0 \quad \forall \boldsymbol{\varphi}, \boldsymbol{\psi} \in \widehat{\boldsymbol{\Lambda}}_{\nu}
$$

Proof. Proceeding as in the previous proof we have

$$
\begin{aligned}
\left\langle S_{1}(\boldsymbol{\varphi})-S_{1}(\boldsymbol{\psi}), \boldsymbol{\varphi}-\boldsymbol{\psi}\right\rangle= & d_{1}\left(\mathcal{V}_{1} \boldsymbol{\varphi} ; \mathcal{V}_{1} \boldsymbol{\varphi}, \mathcal{V}_{1}(\boldsymbol{\varphi}-\boldsymbol{\psi})\right)-d_{1}\left(\mathcal{V}_{1} \boldsymbol{\psi} ; \mathcal{V}_{1} \boldsymbol{\psi}, \mathcal{V}_{1}(\boldsymbol{\varphi}-\boldsymbol{\psi})\right) \\
= & \nu a_{1}\left(\mathcal{V}_{1} \boldsymbol{\varphi}-\mathcal{V}_{1} \boldsymbol{\psi}, \mathcal{V}_{1}(\boldsymbol{\varphi}-\boldsymbol{\psi})\right) \\
& +\frac{1}{2}\left(e_{1}\left(\mathcal{V}_{1} \boldsymbol{\varphi} ; \mathcal{V}_{1} \boldsymbol{\varphi}, \mathcal{V}_{1}(\boldsymbol{\varphi}-\boldsymbol{\psi})\right)-e_{1}\left(\mathcal{V}_{1} \boldsymbol{\varphi} ; \mathcal{V}_{1}(\boldsymbol{\varphi}-\boldsymbol{\psi}), \mathcal{V}_{1} \boldsymbol{\varphi}\right)\right) \\
& +\frac{1}{2}\left(e_{1}\left(\mathcal{V}_{1} \boldsymbol{\psi} ; \mathcal{V}_{1} \boldsymbol{\psi}, \mathcal{V}_{1}(\boldsymbol{\varphi}-\boldsymbol{\psi})\right)-e_{1}\left(\mathcal{V}_{1} \boldsymbol{\psi} ; \mathcal{V}_{1}(\boldsymbol{\varphi}-\boldsymbol{\psi}), \mathcal{V}_{1} \boldsymbol{\psi}\right)\right)
\end{aligned}
$$

Adding and subtracting $\frac{1}{2} e_{1}\left(\mathcal{V}_{1} \boldsymbol{\varphi} ; \mathcal{V}_{1} \boldsymbol{\psi}, \mathcal{V}_{1}(\boldsymbol{\varphi}-\boldsymbol{\psi})\right)+\frac{1}{2} e_{1}\left(\mathcal{V}_{1} \boldsymbol{\varphi} ; \mathcal{V}_{1}(\boldsymbol{\varphi}-\boldsymbol{\psi}), \mathcal{V}_{1} \boldsymbol{\psi}\right)$ and using the inequalities (26) and (31) we obtain

$$
\left\langle S_{1}(\boldsymbol{\varphi})-S_{1}(\boldsymbol{\psi}), \boldsymbol{\varphi}-\boldsymbol{\psi}\right\rangle \geq\left(\frac{\nu}{C_{0}^{2}}-C_{2}\left\|\mathcal{V}_{1} \boldsymbol{\varphi}\right\|_{1, \Omega_{1}}-C_{2}\left\|\mathcal{V}_{1} \boldsymbol{\psi}\right\|_{1, \Omega_{1}}\right)\left\|\mathcal{V}_{1} \boldsymbol{\varphi}-\mathcal{V}_{1} \boldsymbol{\psi}\right\|_{1, \Omega_{1}}\left\|\mathcal{V}_{1}(\boldsymbol{\varphi}-\boldsymbol{\psi})\right\|_{1, \Omega_{1}}
$$

Now (61) follows by using Lemma 4.1 and the definition (59) of $\widehat{\boldsymbol{\Lambda}}_{\nu}$.

Lemma 5.6. There exists a positive constant $K_{1}$, depending on $\nu, \Omega,\left\|\mathbf{u}_{\infty}\right\|_{L^{\infty}\left(\Omega_{2}\right)}$ and $d$, such that

$$
\|S(\boldsymbol{\varphi})-S(\boldsymbol{\psi})\|_{\Lambda^{\prime}} \leq K_{1}\|\boldsymbol{\varphi}-\boldsymbol{\psi}\|_{\Lambda} \quad \forall \boldsymbol{\varphi}, \boldsymbol{\psi} \in \widehat{\boldsymbol{\Lambda}}_{\nu}
$$

Proof. We have

$$
\|S(\boldsymbol{\varphi})-S(\boldsymbol{\psi})\|_{\Lambda^{\prime}}:=\sup _{\boldsymbol{\mu} \in \widehat{\boldsymbol{\Lambda}}_{\nu}} \frac{\left|\left\langle S_{1}(\boldsymbol{\varphi})-S_{1}(\boldsymbol{\psi}), \boldsymbol{\mu}\right\rangle+\left\langle S_{2}(\boldsymbol{\varphi}-\boldsymbol{\psi}), \boldsymbol{\mu}\right\rangle\right|}{\|\boldsymbol{\mu}\|_{\Lambda}} .
$$

The thesis follows with $K_{1}=\beta_{2}+\alpha_{2}$ by applying Lemmas 5.3 and 5.4 .

The Dirichlet/Neumann iterative scheme (18)-(20), can be interpreted as a preconditioned Richardson method to solve the Steklov-Poincaré equation (48). Actually, it reads

$$
\begin{aligned}
& \text { given } \boldsymbol{\lambda}^{0} \in \widehat{\Lambda} \\
& \boldsymbol{\lambda}^{k}=\boldsymbol{\lambda}^{k-1}+\theta S_{2}^{-1}\left(\boldsymbol{\chi}\left(\boldsymbol{\lambda}^{k-1}\right)-S\left(\boldsymbol{\lambda}^{k-1}\right)\right), \quad k \geq 1
\end{aligned}
$$


By an induction argument on (20) it can be shown that $\boldsymbol{\lambda}^{k} \in \widehat{\boldsymbol{\Lambda}}$ for all $k \geq 0$.

We introduce the $S_{2}$-scalar product

$$
(\boldsymbol{\psi}, \boldsymbol{\varphi})_{S_{2}}:=\frac{1}{2}\left(\left\langle S_{2} \boldsymbol{\psi}, \boldsymbol{\varphi}\right\rangle+\left\langle S_{2} \boldsymbol{\varphi}, \boldsymbol{\psi}\right\rangle\right) \text { for } \boldsymbol{\varphi}, \boldsymbol{\psi} \in \widehat{\Lambda}
$$

The corresponding $S_{2}$-norm

$$
\|\boldsymbol{\psi}\|_{S_{2}}:=\sqrt{(\boldsymbol{\psi}, \boldsymbol{\psi})_{S_{2}}}=\sqrt{\left\langle S_{2} \boldsymbol{\psi}, \boldsymbol{\psi}\right\rangle} \text { for } \boldsymbol{\psi} \in \widehat{\boldsymbol{\Lambda}}
$$

is equivalent to the norm $\|\cdot\|_{\Lambda}$, for any function $\boldsymbol{\psi} \in \widehat{\Lambda}$. Actually, it satisfies the two-side inequality

$$
\alpha_{1}\|\boldsymbol{\psi}\|_{\Lambda}^{2} \leq\|\boldsymbol{\psi}\|_{S_{2}}^{2} \leq \alpha_{2}\|\boldsymbol{\psi}\|_{\Lambda}^{2} \quad \forall \boldsymbol{\psi} \in \widehat{\boldsymbol{\Lambda}}
$$

where $\alpha_{1}$ and $\alpha_{2}$ are introduced in (57) and (58), respectively.

Given a relaxation parameter $\theta$, consider the following non-linear operators

$$
T_{\theta}: \widehat{\boldsymbol{\Lambda}} \longrightarrow \widehat{\boldsymbol{\Lambda}}, \quad \boldsymbol{\psi} \longmapsto T_{\theta}(\boldsymbol{\psi}):=-\theta S_{2}^{-1} S_{1}(\boldsymbol{\psi})+(1-\theta) \boldsymbol{\psi}
$$

and

$$
G_{\theta}: \widehat{\Lambda} \longrightarrow \widehat{\Lambda}, \quad \boldsymbol{\psi} \longmapsto G_{\theta}(\boldsymbol{\psi}):=\theta S_{2}^{-1} \chi(\boldsymbol{\psi})
$$

Then (62) reads

$$
\begin{aligned}
& \text { given } \boldsymbol{\lambda}^{0} \in \widehat{\boldsymbol{\Lambda}} \\
& \boldsymbol{\lambda}^{k}=\left(T_{\theta}+G_{\theta}\right)\left(\boldsymbol{\lambda}^{k-1}\right), \quad k \geq 1
\end{aligned}
$$

In order to prove the convergence of the sequence $\boldsymbol{\lambda}^{k}$ to the solution of (48), it is sufficient to prove that $T_{\theta}+G_{\theta}$ is a contraction with respect to the $S_{2}$-norm.

When $\mathbf{f}=\mathbf{0}, T_{\theta}+G_{\theta} \equiv T_{\theta}$; thus we begin to prove that the map $T_{\theta}$ is a contraction with respect to the $S_{2}$-norm.

\subsection{Contractivity of the operator $T_{\theta}$}

Theorem 5.7. If $\left\|\mathbf{u}_{\infty}\right\|_{L^{\infty}\left(\Omega_{2}\right)}$ is sufficiently small, then there exist two positive constants $\tilde{\theta} \in(0,1]$ and $K_{\theta} \in$ $(0,1)$ such that

$$
T_{\theta}(\boldsymbol{\lambda}) \in \widehat{\boldsymbol{\Lambda}}_{\nu}, \quad \forall \boldsymbol{\lambda} \in \widehat{\boldsymbol{\Lambda}}_{\nu}, \quad \forall \theta \in(0, \tilde{\theta}) .
$$

Moreover

$$
\left\|T_{\theta}(\boldsymbol{\varphi})-T_{\theta}(\boldsymbol{\psi})\right\|_{S_{2}} \leq K_{\theta}\|\boldsymbol{\varphi}-\boldsymbol{\psi}\|_{S_{2}} \quad \forall \boldsymbol{\varphi}, \boldsymbol{\psi} \in \widehat{\boldsymbol{\Lambda}}_{\nu}
$$

i.e. $T_{\theta}$ is a contraction in $\widehat{\mathbf{\Lambda}}_{\nu}$.

Proof. We remark that

$$
T_{\theta}(\boldsymbol{\psi})=-\theta S_{2}^{-1} S_{1}(\boldsymbol{\psi})+(1-\theta) \boldsymbol{\psi}=\boldsymbol{\psi}-\theta S_{2}^{-1} S(\boldsymbol{\psi})
$$


By the definition (63) we obtain

$$
\begin{aligned}
\left\|T_{\theta}(\boldsymbol{\varphi})-T_{\theta}(\boldsymbol{\psi})\right\|_{S_{2}}^{2}= & \left\langle S_{2} T_{\theta}(\boldsymbol{\varphi})-S_{2} T_{\theta}(\boldsymbol{\psi}), T_{\theta}(\boldsymbol{\varphi})-T_{\theta}(\boldsymbol{\psi})\right\rangle \\
= & \|\boldsymbol{\varphi}-\boldsymbol{\psi}\|_{S_{2}}^{2}-\theta\left\langle S_{2}(\boldsymbol{\varphi}-\boldsymbol{\psi}), S_{2}^{-1}(S(\boldsymbol{\varphi})-S(\boldsymbol{\psi}))\right\rangle \\
& -\theta\langle S(\boldsymbol{\varphi})-S(\boldsymbol{\psi}), \boldsymbol{\varphi}-\boldsymbol{\psi}\rangle \\
& +\theta^{2}\left\langle S(\boldsymbol{\varphi})-S(\boldsymbol{\psi}), S_{2}^{-1}(S(\boldsymbol{\varphi})-S(\boldsymbol{\psi}))\right\rangle
\end{aligned}
$$

and setting $\boldsymbol{\mu}_{\varphi}=S_{2}^{-1} S(\boldsymbol{\varphi})$ and $\boldsymbol{\mu}_{\psi}=S_{2}^{-1} S(\boldsymbol{\psi})$ we can write

$$
\begin{aligned}
\left\|T_{\theta}(\boldsymbol{\varphi})-T_{\theta}(\boldsymbol{\psi})\right\|_{S_{2}}^{2}= & (1-2 \theta)\|\boldsymbol{\varphi}-\boldsymbol{\psi}\|_{S_{2}}^{2} \\
& -\theta\left[\left\langle S_{2}(\boldsymbol{\varphi}-\boldsymbol{\psi}), \boldsymbol{\mu}_{\varphi}-\boldsymbol{\mu}_{\psi}\right\rangle-\left\langle S_{2}\left(\boldsymbol{\mu}_{\varphi}-\boldsymbol{\mu}_{\psi}\right), \boldsymbol{\varphi}-\boldsymbol{\psi}\right\rangle\right] \\
& -2 \theta\left\langle S_{1}(\boldsymbol{\varphi})-S_{1}(\boldsymbol{\psi}), \boldsymbol{\varphi}-\boldsymbol{\psi}\right\rangle \\
& +\theta^{2}\left\langle S(\boldsymbol{\varphi})-S(\boldsymbol{\psi}), S_{2}^{-1}(S(\boldsymbol{\varphi})-S(\boldsymbol{\psi}))\right\rangle .
\end{aligned}
$$

We examine the second and the fourth terms separately.

The skew-symmetric part of $S_{2}$ can be bounded as follows: there exists a positive constant $K_{2}=K_{2}\left(\nu, \Omega_{2},\left\|\mathbf{u}_{\infty}\right\|_{L^{\infty}\left(\Omega_{2}\right)}, d\right)$ such that

$$
\left|\left\langle S_{2}(\boldsymbol{\varphi}-\boldsymbol{\psi}), \boldsymbol{\mu}_{\varphi}-\boldsymbol{\mu}_{\psi}\right\rangle-\left\langle S_{2}\left(\boldsymbol{\mu}_{\varphi}-\boldsymbol{\mu}_{\psi}\right), \boldsymbol{\varphi}-\boldsymbol{\psi}\right\rangle\right| \leq \frac{K_{2}}{\alpha_{1}^{2}}\|\boldsymbol{\varphi}-\boldsymbol{\psi}\|_{S_{2}}^{2}, \quad \forall \boldsymbol{\varphi}, \boldsymbol{\psi} \in \widehat{\boldsymbol{\Lambda}}_{\nu}
$$

Indeed, by (41) and Lemma 4.7 we have

$$
\begin{aligned}
\left|\left\langle S_{2}(\boldsymbol{\varphi}-\boldsymbol{\psi}), \boldsymbol{\mu}_{\varphi}-\boldsymbol{\mu}_{\psi}\right\rangle-\left\langle S_{2}\left(\boldsymbol{\mu}_{\varphi}-\boldsymbol{\mu}_{\psi}\right), \boldsymbol{\varphi}-\boldsymbol{\psi}\right\rangle\right| \\
=\left|d_{2}\left(\mathbf{u}_{\infty} ; \mathcal{O}_{2}(\boldsymbol{\varphi}-\boldsymbol{\psi}), \mathcal{O}_{2}\left(\boldsymbol{\mu}_{\varphi}-\boldsymbol{\mu}_{\psi}\right)\right)-d_{2}\left(\mathbf{u}_{\infty} ; \mathcal{O}_{2}\left(\boldsymbol{\mu}_{\varphi}-\boldsymbol{\mu}_{\psi}\right), \mathcal{O}_{2}(\boldsymbol{\varphi}-\boldsymbol{\psi})\right)\right| \\
=\mid a_{2}\left(\mathcal{O}_{2}(\boldsymbol{\varphi}-\boldsymbol{\psi}), \mathcal{O}_{2}\left(\boldsymbol{\mu}_{\varphi}-\boldsymbol{\mu}_{\psi}\right)\right)-a_{2}\left(\mathcal{O}_{2}\left(\boldsymbol{\mu}_{\varphi}-\boldsymbol{\mu}_{\psi}\right), \mathcal{O}_{2}(\boldsymbol{\varphi}-\boldsymbol{\psi})\right) \\
\quad+e_{2}\left(\mathbf{u}_{\infty} ; \mathcal{O}_{2}(\boldsymbol{\varphi}-\boldsymbol{\psi}), \mathcal{O}_{2}\left(\boldsymbol{\mu}_{\varphi}-\boldsymbol{\mu}_{\psi}\right)\right)-e_{2}\left(\mathbf{u}_{\infty} ; \mathcal{O}_{2}\left(\boldsymbol{\mu}_{\varphi}-\boldsymbol{\mu}_{\psi}\right), \mathcal{O}_{2}(\boldsymbol{\varphi}-\boldsymbol{\psi})\right) \mid \\
\leq 2 C_{3}\left\|\mathbf{u}_{\infty}\right\|_{L^{\infty}\left(\Omega_{2}\right)}\left\|\mathcal{O}_{2}(\boldsymbol{\varphi}-\boldsymbol{\psi})\right\|_{1, \Omega_{2}}\left\|\mathcal{O}_{2}\left(\boldsymbol{\mu}_{\varphi}-\boldsymbol{\mu}_{\psi}\right)\right\|_{1, \Omega_{2}} \\
\leq 2 C_{3} C_{\beta}^{2}\left\|\mathbf{u}_{\infty}\right\|_{L^{\infty}\left(\Omega_{2}\right)}\|\boldsymbol{\varphi}-\boldsymbol{\psi}\|_{\Lambda}\left\|\boldsymbol{\mu}_{\varphi}-\boldsymbol{\mu}_{\psi}\right\|_{\Lambda}
\end{aligned}
$$

Moreover, from Lemma 5.3 it follows that the inverse of $S_{2}$ exists and satisfies

$$
\left\|S_{2}^{-1}\right\|_{*}:=\sup _{\boldsymbol{\zeta} \in \widehat{\Lambda}^{\prime}} \frac{\left\|S_{2}^{-1} \boldsymbol{\zeta}\right\|_{\Lambda}}{\|\boldsymbol{\zeta}\|_{\Lambda^{\prime}}} \leq \frac{1}{\alpha_{1}}
$$

Then by Lemma 5.6 it holds

$$
\left\|\boldsymbol{\mu}_{\varphi}-\boldsymbol{\mu}_{\psi}\right\|_{\Lambda} \leq\left\|S_{2}^{-1}\right\|_{*}\|S(\boldsymbol{\varphi})-S(\boldsymbol{\psi})\|_{\Lambda^{\prime}} \leq \frac{1}{\alpha_{1}} K_{1}\|\boldsymbol{\varphi}-\boldsymbol{\psi}\|_{\Lambda}
$$

Finally, by (64) we have

$$
\left|\left\langle S_{2}(\boldsymbol{\varphi}-\boldsymbol{\psi}), \boldsymbol{\mu}_{\varphi}-\boldsymbol{\mu}_{\psi}\right\rangle-\left\langle S_{2}\left(\boldsymbol{\mu}_{\varphi}-\boldsymbol{\mu}_{\psi}\right), \boldsymbol{\varphi}-\boldsymbol{\psi}\right\rangle\right| \leq \frac{2}{\alpha_{1}^{2}} C_{3} C_{\beta}^{2} K_{1}\left\|\mathbf{u}_{\infty}\right\|_{L^{\infty}\left(\Omega_{2}\right)}\|\boldsymbol{\varphi}-\boldsymbol{\psi}\|_{S_{2}}^{2}
$$


thus (67) is satisfied with $K_{2}=2 C_{3} C_{\beta}^{2} K_{1}\left\|\mathbf{u}_{\infty}\right\|_{L^{\infty}\left(\Omega_{2}\right)}$.

Using again Lemma 5.6, (64) and (68) we obtain

$$
\left\langle S(\boldsymbol{\varphi})-S(\boldsymbol{\psi}), S_{2}^{-1}(S(\boldsymbol{\varphi})-S(\boldsymbol{\psi}))\right\rangle \leq\left\|S_{2}^{-1}\right\|_{*}\|S(\boldsymbol{\varphi})-S(\boldsymbol{\psi})\|_{\Lambda^{\prime}}^{2} \leq \frac{K_{1}^{2}}{\alpha_{1}}\|\boldsymbol{\varphi}-\boldsymbol{\psi}\|_{\Lambda}^{2}
$$

and therefore

$$
\left\|T_{\theta}(\boldsymbol{\varphi})-T_{\theta}(\boldsymbol{\psi})\right\|_{S_{2}}^{2} \leq\left(1+\theta\left(\frac{K_{2}}{\alpha_{1}^{2}}-2\right)+\theta^{2} \frac{K_{1}^{2}}{\alpha_{1}^{2}}\right)\|\boldsymbol{\varphi}-\boldsymbol{\psi}\|_{S_{2}}^{2}
$$

If $K_{2}-2 \alpha_{1}^{2}<0$ and if we choose $\theta \in(0, \widetilde{\theta})$, with

$$
\widetilde{\theta}=\frac{2 \alpha_{1}^{2}-K_{2}}{K_{1}^{2}}
$$

then

$$
K_{\theta}:=\left(1+\theta\left(\frac{K_{2}}{\alpha_{1}^{2}}-2\right)+\theta^{2} \frac{K_{1}^{2}}{\alpha_{1}^{2}}\right)^{1 / 2}<1 .
$$

Furthermore, since $T_{\theta}(\mathbf{0})=\mathbf{0}$, directly from (66) one has

$$
\left\|T_{\theta}(\boldsymbol{\lambda})\right\|_{S_{2}} \leq K_{\theta}\|\boldsymbol{\lambda}\|_{S_{2}} \quad \forall \boldsymbol{\lambda} \in \widehat{\boldsymbol{\Lambda}}_{\nu} \quad \text { with } \quad K_{\theta}<1
$$

Consequently we conclude that $T_{\theta}(\boldsymbol{\lambda}) \in \widehat{\boldsymbol{\Lambda}}_{\nu}, \forall \boldsymbol{\lambda} \in \widehat{\boldsymbol{\Lambda}}_{\nu}$.

Remark 5.8. Bearing in mind that $K_{2}$ depends on both $\left\|\mathbf{u}_{\infty}\right\|_{L^{\infty}\left(\Omega_{2}\right)}$ and $\nu$, and that $\alpha_{1}$ depends on $\nu$, the condition $K_{2}-2 \alpha_{1}^{2}<0$ is in fact an implicit condition on the smallness of $\left\|\mathbf{u}_{\infty}\right\|_{L^{\infty}\left(\Omega_{2}\right)}$ with respect to the viscosity coefficient $\nu$.

\subsection{Contractivity of the operator $T_{\theta}+G_{\theta}$}

In the next theorem we prove that the map $G_{\theta}$ is a contraction with respect to the $S_{2}$-norm.

Theorem 5.9. If (37) holds, there exists a positive constant $K_{3}$, depending on $\nu, \Omega, d$ and $\mathbf{f}$ such that, for all $\boldsymbol{\varphi}, \boldsymbol{\psi} \in \widehat{\Lambda}$ satisfying the assumption (23), it holds

$$
\left\|G_{\theta}(\boldsymbol{\varphi})-G_{\theta}(\boldsymbol{\psi})\right\|_{S_{2}}^{2} \leq \theta^{2} \frac{K_{3}^{2}}{\alpha_{1}^{2}}\|\boldsymbol{\varphi}-\boldsymbol{\psi}\|_{S_{2}}^{2} .
$$

Proof. By (68), we have

$$
\begin{aligned}
\left\|G_{\theta}(\boldsymbol{\varphi})-G_{\theta}(\boldsymbol{\psi})\right\|_{S_{2}}^{2} & =\theta^{2}\left\langle\chi(\boldsymbol{\varphi})-\chi(\boldsymbol{\psi}), S_{2}^{-1}(\chi(\boldsymbol{\varphi})-\chi(\boldsymbol{\psi}))\right\rangle \\
& \leq \theta^{2}\left\|S_{2}^{-1}\right\|_{*}\|\chi(\boldsymbol{\varphi})-\chi(\boldsymbol{\psi})\|_{\Lambda^{\prime}}^{2} \leq \frac{\theta^{2}}{\alpha_{1}}\|\chi(\boldsymbol{\varphi})-\chi(\boldsymbol{\psi})\|_{\Lambda^{\prime}}^{2}
\end{aligned}
$$


In order to estimate $|\langle\boldsymbol{\chi}(\boldsymbol{\varphi})-\boldsymbol{\chi}(\boldsymbol{\psi}), \boldsymbol{\mu}\rangle|$ we use the definition (56) and we add and subtract $\frac{1}{2}\left[e_{1}\left(\mathcal{V}_{1}^{*} \boldsymbol{\varphi} ; \mathcal{V}_{1} \boldsymbol{\psi}, \mathcal{V}_{1} \boldsymbol{\mu}\right)\right.$ $\left.+e_{1}\left(\mathcal{V}_{1}^{*} \boldsymbol{\varphi} ; \mathcal{V}_{1} \boldsymbol{\mu}, \mathcal{V}_{1} \boldsymbol{\psi}\right)+e_{1}\left(\mathcal{V}_{1} \boldsymbol{\psi}-\mathcal{V}_{1}^{*} \boldsymbol{\psi} ; \mathcal{V}_{1}^{*} \boldsymbol{\varphi}, \mathcal{V}_{1} \boldsymbol{\mu}\right)+e_{1}\left(\mathcal{V}_{1} \boldsymbol{\psi}-\mathcal{V}_{1}^{*} \boldsymbol{\psi} ; \mathcal{V}_{1} \boldsymbol{\mu}, \mathcal{V}_{1}^{*} \boldsymbol{\varphi}\right)\right]$. Then we obtain

$$
\begin{aligned}
|\langle\chi(\boldsymbol{\varphi})-\chi(\boldsymbol{\psi}), \boldsymbol{\mu}\rangle| \leq & \left\|\mathcal{V}_{1} \boldsymbol{\mu}\right\|_{1, \Omega_{1}}\left[\nu\left\|\mathcal{V}_{1}^{*} \boldsymbol{\varphi}-\mathcal{V}_{1}^{*} \boldsymbol{\psi}\right\|_{1, \Omega_{1}}\right. \\
& +2 C_{2}\left(\left\|\mathcal{V}_{1}^{*} \boldsymbol{\varphi}\right\|_{1, \Omega_{1}}\left\|\mathcal{V}_{1} \boldsymbol{\varphi}-\mathcal{V}_{1} \boldsymbol{\psi}\right\|_{1, \Omega_{1}}+\left\|\mathcal{V}_{1} \boldsymbol{\psi}\right\|_{1, \Omega_{1}}\left\|\mathcal{V}_{1}^{*} \boldsymbol{\varphi}-\mathcal{V}_{1}^{*} \boldsymbol{\psi}\right\|_{1, \Omega_{1}}\right) \\
& \left.+C_{2}\left(\left\|\mathcal{V}_{1}^{*} \boldsymbol{\varphi}-\mathcal{V}_{1}^{*} \boldsymbol{\psi}\right\|_{1, \Omega_{1}}\left\|\mathcal{V}_{1}^{*} \boldsymbol{\varphi}\right\|_{1, \Omega_{1}}+\left\|\mathcal{V}_{1}^{*} \boldsymbol{\varphi}-\mathcal{V}_{1}^{*} \boldsymbol{\psi}\right\|_{1, \Omega_{1}}\left\|\mathcal{V}_{1}^{*} \boldsymbol{\psi}\right\|_{1, \Omega_{1}}\right)\right]
\end{aligned}
$$

Applying Lemmas 4.1, 4.4, 4.5 and 4.6, we have

$$
\|\chi(\varphi)-\chi(\psi)\|_{\Lambda^{\prime}} \leq K_{3}\|\varphi-\psi\|_{\Lambda}
$$

with $K_{3}=\frac{3}{2} C_{1}\left[\nu C_{\gamma}^{*}\left(1+\frac{1}{C_{0}^{2}}\right)+2 C_{2} C_{\alpha}^{*}\|\mathbf{f}\|_{-1, \Omega_{1}}\left(C_{\gamma}+C_{\gamma}^{*}\right)\right]$, whence (71).

Theorem 5.10. If $\left\|\mathbf{u}_{\infty}\right\|_{L^{\infty}\left(\Omega_{2}\right)}$ is sufficiently small and if (37) is satisfied, then there exist two positive constants $\tilde{\theta}^{*} \in(0,1]$ and $K_{\theta^{*}} \in(0,1)$ such that

$$
T_{\theta}(\boldsymbol{\lambda})+G_{\theta}(\boldsymbol{\lambda}) \in \widehat{\boldsymbol{\Lambda}}_{\nu}, \quad \forall \boldsymbol{\lambda} \in \widehat{\boldsymbol{\Lambda}}_{\nu}, \quad \forall \theta \in\left(0, \tilde{\theta}^{*}\right) .
$$

Moreover

$$
\left\|\left(T_{\theta}+G_{\theta}\right)(\boldsymbol{\varphi})-\left(T_{\theta}+G_{\theta}\right)(\boldsymbol{\psi})\right\|_{S_{2}} \leq K_{\theta^{*}}\|\boldsymbol{\varphi}-\boldsymbol{\psi}\|_{S_{2}} \quad \forall \boldsymbol{\varphi}, \boldsymbol{\psi} \in \widehat{\boldsymbol{\Lambda}}_{\nu},
$$

i.e. $T_{\theta}+G_{\theta}$ is a contraction in $\widehat{\boldsymbol{\Lambda}}_{\nu}$.

Proof. From the definitions of the $S_{2}$-norm and the $S_{2}$-inner product we have:

$$
\begin{aligned}
\left\|T_{\theta}(\boldsymbol{\varphi})-T_{\theta}(\boldsymbol{\psi})+G_{\theta}(\boldsymbol{\varphi})-G_{\theta}(\boldsymbol{\psi})\right\|_{S_{2}}^{2} \leq & \left\|T_{\theta}(\boldsymbol{\varphi})-T_{\theta}(\boldsymbol{\psi})\right\|_{S_{2}}^{2}+\left\|G_{\theta}(\boldsymbol{\varphi})-G_{\theta}(\boldsymbol{\psi})\right\|_{S_{2}}^{2} \\
& +2\left(T_{\theta}(\boldsymbol{\varphi})-T_{\theta}(\boldsymbol{\psi}), G_{\theta}(\boldsymbol{\varphi})-G_{\theta}(\boldsymbol{\psi})\right)_{S_{2}} .
\end{aligned}
$$

Setting $\boldsymbol{\mu}_{\varphi}=\theta S_{2}^{-1} S(\boldsymbol{\varphi})$ and $\boldsymbol{\mu}_{\psi}=\theta S_{2}^{-1} S(\boldsymbol{\psi})$ we can write

$$
\begin{aligned}
& 2\left(T_{\theta}(\boldsymbol{\varphi})-T_{\theta}(\boldsymbol{\psi}), G_{\theta}(\boldsymbol{\varphi})-G_{\theta}(\boldsymbol{\psi})\right)_{S_{2}} \\
&=\quad \theta\left\langle S_{2}(\boldsymbol{\varphi}-\boldsymbol{\psi})-\theta(S(\boldsymbol{\varphi})-S(\boldsymbol{\psi})), \theta S_{2}^{-1}(\boldsymbol{\chi}(\boldsymbol{\varphi})-\boldsymbol{\chi}(\boldsymbol{\psi}))\right\rangle \\
&+\theta\left\langle\boldsymbol{\chi}(\boldsymbol{\varphi})-\chi(\boldsymbol{\psi}), \boldsymbol{\varphi}-\boldsymbol{\psi}-\theta S_{2}^{-1}(S(\boldsymbol{\varphi})-S(\boldsymbol{\psi}))\right\rangle \\
&= \theta\left\langle S_{2}\left(\boldsymbol{\varphi}-\boldsymbol{\mu}_{\varphi}-\boldsymbol{\psi}+\boldsymbol{\mu}_{\psi}\right), S_{2}^{-1}(\boldsymbol{\chi}(\boldsymbol{\varphi})-\boldsymbol{\chi}(\boldsymbol{\psi})\rangle\right. \\
&+\theta\left\langle\boldsymbol{\chi}(\boldsymbol{\varphi})-\boldsymbol{\chi}(\boldsymbol{\psi}), \boldsymbol{\varphi}-\boldsymbol{\mu}_{\varphi}-\boldsymbol{\psi}+\boldsymbol{\mu}_{\psi}\right\rangle \\
& \leq \theta\left(\frac{\alpha_{2}}{\alpha_{1}}+1\right)\left\|\boldsymbol{\varphi}-\boldsymbol{\mu}_{\varphi}-\boldsymbol{\psi}+\boldsymbol{\mu}_{\psi}\right\|_{\Lambda}\|\chi(\boldsymbol{\varphi})-\chi(\boldsymbol{\psi})\|_{\Lambda^{\prime}} .
\end{aligned}
$$

By the triangle inequality and (69) we have

$$
\left\|\boldsymbol{\varphi}-\boldsymbol{\mu}_{\varphi}-\boldsymbol{\psi}+\boldsymbol{\mu}_{\psi}\right\|_{\Lambda} \leq\|\boldsymbol{\varphi}-\boldsymbol{\psi}\|_{\Lambda}+\left\|\boldsymbol{\mu}_{\varphi}-\boldsymbol{\mu}_{\psi}\right\|_{\Lambda} \leq\left(1+\theta \frac{K_{1}}{\alpha_{1}}\right)\|\boldsymbol{\varphi}-\boldsymbol{\psi}\|_{\Lambda}
$$


and by (72) we can conclude that

$$
2\left(T_{\theta}(\boldsymbol{\varphi})-T_{\theta}(\boldsymbol{\psi}), G_{\theta}(\boldsymbol{\varphi})-G_{\theta}(\boldsymbol{\psi})\right)_{S_{2}} \leq\left[\theta \frac{K_{4}}{\alpha_{1}^{2}}+\theta^{2} \frac{K_{4} K_{1}}{\alpha_{1}^{3}}\right]\|\boldsymbol{\varphi}-\boldsymbol{\psi}\|_{S_{2}}^{2},
$$

where $K_{4}=K_{3}\left(\alpha_{2}+\alpha_{1}\right)$. Now (73) follows.

By Theorems 5.7 and 5.9 , with

$$
K_{\theta^{*}}=\left(1+\theta\left(\frac{K_{2}}{\alpha_{1}^{2}}-2+\frac{K_{4}}{\alpha_{1}^{2}}\right)+\theta^{2}\left(\frac{K_{1}}{\alpha_{1}^{2}}+\frac{K_{1} K_{4}}{\alpha_{1}^{3}}\right)\right)^{1 / 2}
$$

we have $K_{\theta}^{*}<1$ if $K_{2}-2 \alpha_{1}^{2}+K_{4}<0$ and if we choose $\theta \in\left(0, \widetilde{\theta}^{*}\right)$, with

$$
\widetilde{\theta}^{*}=\frac{2 \alpha_{1}^{2}-K_{2}-K_{4}}{K_{1}^{2}+K_{1} K_{4} / \alpha_{1}}
$$

Furthermore, since $T_{\theta}(\mathbf{0})+G_{\theta}(\mathbf{0})=\mathbf{0}$, directly from (73) one has

$$
\left\|T_{\theta}(\boldsymbol{\lambda})+G_{\theta}(\boldsymbol{\lambda})\right\|_{S_{2}} \leq K_{\theta^{*}}\|\boldsymbol{\lambda}\|_{S_{2}} \quad \forall \boldsymbol{\lambda} \in \widehat{\boldsymbol{\Lambda}}_{\nu}
$$

i.e. $T_{\theta}(\boldsymbol{\lambda})+G_{\theta}(\boldsymbol{\lambda}) \in \widehat{\boldsymbol{\Lambda}}_{\nu}, \forall \boldsymbol{\lambda} \in \widehat{\boldsymbol{\Lambda}}_{\nu}$.

\section{An existence and uniqueness result for the Coupled Navier-Stokes/Oseen PROBLEM}

On the ground of the convergence results of the previous sections, we can infer the existence of a solution of the coupled Navier-Stokes/Oseen problem (16). This proof of existence is alternative to that proposed by Feistauer and Schwab in [5].

When considering problem (16), we limit ourselves to prove the existence of the velocity fields $\mathbf{u}_{1}$ and $\mathbf{u}_{2}$. The existence of $p_{1}$ and $p_{2}$ then follows by classical arguments (see e.g. [14]).

Theorem 6.1. Under the assumptions of Theorem 5.10 there exists a unique solution of the coupled problem (16).

Proof. From Theorem 5.10 we know that the sequence $\boldsymbol{\lambda}^{k}=\mathbf{u}_{1 \mid \Gamma}^{k}$ is convergent in $\widehat{\boldsymbol{\Lambda}}_{\nu}$ for $0<\theta<\widetilde{\theta}^{*}$. Then it is a Cauchy sequence in $\widehat{\boldsymbol{\Lambda}}_{\nu}$. Thanks to Lemma 4.4 and Lemma $4.6, \mathbf{u}_{1}^{k}$ is a Cauchy sequence in $\left[V_{1}\right]^{d}$, and since $\left[V_{1}\right]^{d}$ is a Hilbert space, it is a convergent sequence in $\left[V_{1}\right]^{d}$.

From (20) we deduce that

$$
\lim _{k \rightarrow \infty} \mathbf{u}_{2 \mid \Gamma}^{k}=\lim _{k \rightarrow \infty} \mathbf{u}_{1 \mid \Gamma}^{k},
$$

then, using again Lemma 4.4 and Lemma 4.6 , we deduce that $\mathbf{u}_{2}^{k}$ is also a convergence sequence in $\left[V_{2}\right]^{d}$.

It follows that there exist $\left(\tilde{\mathbf{u}}_{1}, \tilde{p}_{1}\right) \in\left[V_{1}\right]^{d} \times L^{2}\left(\Omega_{1}\right)$ and $\left(\tilde{\mathbf{u}}_{2}, \tilde{p}_{2}\right) \in\left[V_{2}\right]^{d} \times L^{2}\left(\Omega_{2}\right)$ such that

$$
\lim _{k \rightarrow \infty}\left\|\mathbf{u}_{i}^{k}-\tilde{\mathbf{u}}_{i}\right\|_{1, \Omega_{i}}=0, \quad \lim _{k \rightarrow \infty}\left\|p_{i}^{k}-\tilde{p}_{i}\right\|_{0, \Omega_{i}}=0, \quad i=1,2
$$

To prove that $\left(\tilde{\mathbf{u}}_{1}, \tilde{p}_{1}\right)$ and $\left(\tilde{\mathbf{u}}_{2}, \tilde{p}_{2}\right)$ satisfy problem (16), we take the limit in (18) and (19). We omit the analysis on the linear forms $d_{2}$ and $b_{i}(i=1,2)$, but we prove that

$$
\lim _{k \rightarrow \infty} d_{1}\left(\mathbf{u}_{1}^{k} ; \mathbf{u}_{1}^{k}, \mathbf{v}_{1}\right)=d_{1}\left(\tilde{\mathbf{u}}_{1} ; \tilde{\mathbf{u}}_{1}, \mathbf{v}_{1}\right) .
$$


Owing to (31), we have

$$
\begin{aligned}
\left|d_{1}\left(\tilde{\mathbf{u}}_{1} ; \tilde{\mathbf{u}}_{1}, \mathbf{v}_{1}\right)-d_{1}\left(\mathbf{u}_{1}^{k} ; \mathbf{u}_{1}^{k}, \mathbf{v}_{1}\right)\right| & \leq \nu\left|a_{1}\left(\tilde{\mathbf{u}}_{1}-\mathbf{u}_{1}^{k}, \mathbf{v}_{1}\right)\right|+\left|e_{1}\left(\tilde{\mathbf{u}}_{1}-\mathbf{u}_{1}^{k} ; \tilde{\mathbf{u}}_{1}, \mathbf{v}_{1}\right)+e_{1}\left(\mathbf{u}_{1}^{k} ; \tilde{\mathbf{u}}_{1}-\mathbf{u}_{1}^{k}, \mathbf{v}_{1}\right)\right| \\
& \leq\left\|\tilde{\mathbf{u}}_{1}-\mathbf{u}_{1}^{k}\right\|_{1, \Omega_{1}}\left\|\mathbf{v}_{1}\right\|_{1, \Omega_{1}}\left(\nu+C_{2}\left\|\tilde{\mathbf{u}}_{1}\right\|_{1, \Omega_{1}}+C_{2}\left\|\mathbf{u}_{1}^{k}\right\|_{1, \Omega_{1}}\right) .
\end{aligned}
$$

Then from (75) we conclude that

$$
\left|d_{1}\left(\tilde{\mathbf{u}}_{1} ; \tilde{\mathbf{u}}_{1}, \mathbf{v}_{1}\right)-d_{1}\left(\mathbf{u}_{1}^{k} ; \mathbf{u}_{1}^{k}, \mathbf{v}_{1}\right)\right| \rightarrow 0, \quad k \rightarrow \infty
$$

namely (76).

We observe that the continuity of the Oseen flux (11) is guaranteed by the fact that the sequence $\boldsymbol{\lambda}^{k}$ defined in (62) is convergent.

In conclusion, $\left(\tilde{\mathbf{u}}_{i}, \tilde{p}_{i}\right), i=1,2$ provide a solution of problem (9)-(12).

The uniqueness of this solution is a direct consequence of the contraction Theorem 5.10 as well as of the smallness of $\mathbf{f}$ and largeness of $\nu$, our working assumptions. In fact, Theorem 5.10 ensures the uniqueness of the trace of the solutions on $\Gamma$. Moreover, the Oseen problem in $\Omega_{2}$ is well-posed, while the uniqueness of the Navier-Stokes problem in $\Omega_{1}$ is guaranteed provided that $\nu$ is sufficiently large (see again [14]).

A more straightforward proof would make use of Theorem 5.2. Actually, since $\left\{\boldsymbol{\lambda}^{k}\right\}$ converges to a unique $\boldsymbol{\lambda}$, from Theorem 5.2 there exists a couple $\left\{\mathbf{u}_{1}, p_{1}\right\},\left\{\mathbf{u}_{2}, p_{2}\right\}$ of solutions to (16). The uniqueness of $\left\{\mathbf{u}_{2}, p_{2}\right\}$ follows now from the uniqueness of the Oseen problem in $\Omega_{2}$ with Dirichlet data $\mathbf{u}_{2}=\boldsymbol{\lambda}_{2}$ on $\Gamma$, whereas that of $\left\{\mathbf{u}_{1}, p_{1}\right\}$ follows owing to the usual assumption of smallness of data.

\section{Test CASES AND NUMERICAL RESUlts}

In order to approximate the solution of the boundary-value problems (18) and (19) with $d=2$ we use the conformal quadrilateral spectral element method $[1] . \mathbb{Q}_{N}-\mathbb{Q}_{N-2}[1,9]$ is considered in order to satisfy the inf-sup condition [11] and, consequently, to avoid spurious modes on the pressure. Two staggered grids are used for the velocity and the pressure. The first grid is based on the Gauss-Lobatto Legendre nodes (GLL), while the second one is based on the Gauss Legendre nodes (GL). Let us denote by $N$ the polynomial degree used to interpolate the velocity field along both the $x$ and $y$ coordinates. In each spectral element we have $(N+1)^{2}$ GLL nodes and $(N-1)^{2}$ GL nodes; $H$ will denote the maximum side of the spectral elements.

We solve the non-linear Navier Stokes equations in $\Omega_{1}$ by the Newton method, while the linear system associated to the Jacobian matrix as well as the one associated to the Oseen equations in $\Omega_{2}$ are solved by a Bi-CGStab algorithm [16], preconditioned by an incomplete LU factorization.

The relaxation parameter $\theta$ appearing in (20) can either be assigned a priori, or be chosen dynamically so as to minimize the interface error at each step.

In order to test the convergence of our Dirichlet/Neumann $(\mathrm{D} / \mathrm{N})$ algorithm we check that

$$
\max _{i=1,2}\left[\frac{\left\|\mathbf{u}_{i}^{k}-\mathbf{u}_{i}^{k-1}\right\|_{H^{1}\left(\Omega_{i}\right)}}{\left\|\mathbf{u}_{i}^{k}\right\|_{H^{1}\left(\Omega_{i}\right)}}\right] \leq 5 \times 10^{-6},
$$

where $k$ is the $\mathrm{D} / \mathrm{N}$ iteration counter. 


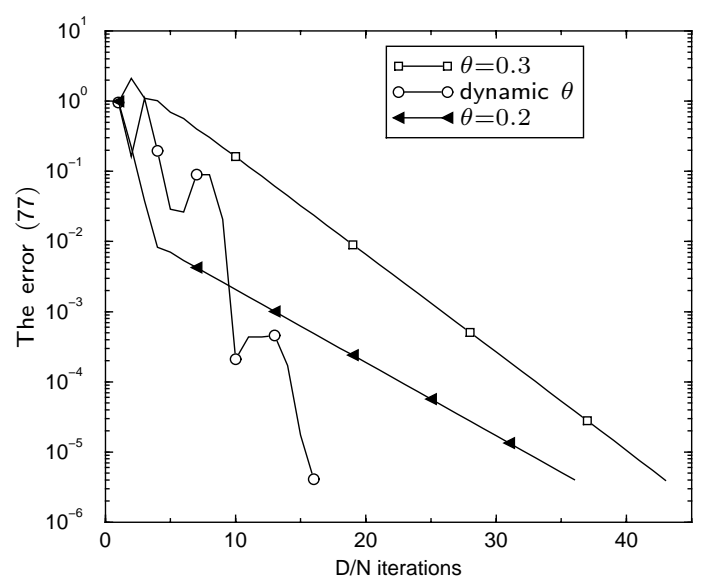

Figure 2. Convergence history of the Dirichlet/Neumann algorithm for the approximation of the Kovasnay solution (78).

\subsection{The Kovasznay analytical solution}

The Kovasznay solution to the steady Navier-Stokes equations is similar to the two-dimensional flow field behind a periodic array of cylinders [8]. It has the following expression:

$$
\begin{aligned}
u_{1}(x, y) & =1-\mathrm{e}^{\lambda x} \cos (2 \pi y), & u_{2}(x, y) & =\frac{\lambda}{2 \pi} \mathrm{e}^{\lambda x} \sin (2 \pi y) \\
p(x, y) & =-\mathrm{e}^{2 \lambda x} / 2, & \lambda & =\frac{1}{2} \operatorname{Re}-\sqrt{\frac{1}{4} \operatorname{Re}^{2}+4 \pi^{2}},
\end{aligned}
$$

where $\operatorname{Re}=1 / \nu$ is the Reynolds number and $\nu=0.025$. The forcing term is $\mathbf{f}=\mathbf{0}$. The Kovasznay solution can also be recovered by solving a Navier-Stokes/Oseen coupling setting $\mathbf{u}_{\infty}=[1,0]^{t}$, since $\mathbf{u} \rightarrow \mathbf{u}_{\infty}$ when $x \rightarrow \infty$. The computational domain is $\Omega=(-1,3) \times(0.5,2.5)$ and a decomposition in two aligned subdomains $\Omega_{1}, \Omega_{2}$ is considered with interface $\Gamma=\{1\} \times(0.5,2.5)$. On the boundary of $\Omega$ we impose a Dirichlet condition. The initial data $\lambda^{0}$ on the interface is chosen in order to satisfy compatibility conditions on the incompressibility constraint (see [12, Chap. 5]).

In Figure 2 we show the convergence history of the Dirichlet/Neumann algorithm (in terms of the relative error (77)) for two a-priori choices of the relaxation parameter $\theta(\theta=0.2,0.3)$ and for the dynamic choice of $\theta$, where we have chosen $N=5$ and $H=0.5$.

We have observed that the rate of convergence of the Dirichlet/Neumann method is independent of the mesh parameters $N, H$ as well as of the interface position.

\subsection{Stationary 2D channel flow over a backward facing step}

We consider now a domain split in three subdomains, as described in Figure 3.

On the boundary of the computational domain we set the following Dirichlet conditions: a parabolic velocity profile at the inflow boundary, no-slip conditions at fixed walls and the solution $\mathbf{u}_{\text {Stokes }}$ of the Stokes problem corresponding to the same data on the outflow. A null force vector field $\mathbf{f}$ is chosen, while $\mathbf{u}_{\infty}$ is chosen equal

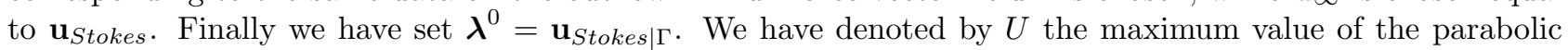
inflow and we have defined the Reynolds number $R e=D U / \nu$, where $D=0.125$ is the step height. We have computed the solution of this test case with both $U=1$ and $U=2$. 


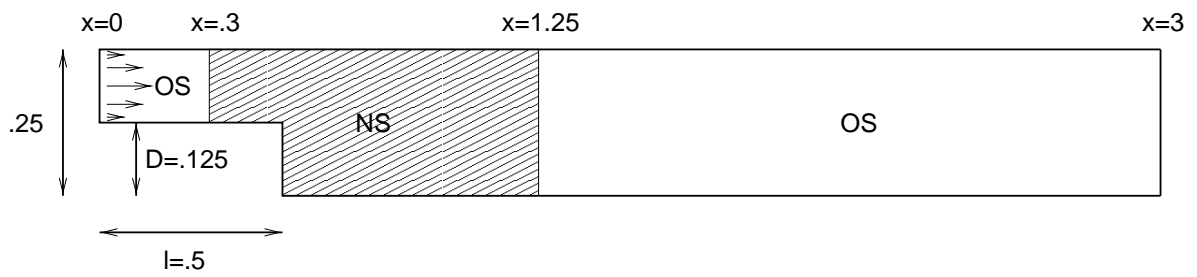

Figure 3. The computational domain for the stationary 2D channel flow over a backward facing step. Three subdomains have been considered: one Navier-Stokes subdomain (grey) and two Oseen subdomains (white).

\begin{tabular}{|l|l|l|c|}
\hline$U=1, \nu=$ & $U=2, \nu=$ & $\operatorname{Re}$ & $\mathrm{D} / \mathrm{N}$ iterations \\
\hline \hline 0.0025 & 0.005 & 50 & 16 \\
$0.001 \overline{6}$ & $0.00 \overline{3}$ & 75 & 17 \\
0.00125 & 0.0025 & 100 & 22 \\
0.001 & 0.002 & 125 & 23 \\
$0.0008 \overline{3}$ & $0.001 \overline{6}$ & 150 & 27 \\
\hline
\end{tabular}

TABLE 1. The D/N iterations for the stationary 2D channel flow over a backward facing step, versus the Reynolds number.

In Table 1 we show the number of Dirichlet/Neumann iterations $\left(I t_{D N}\right)$ needed to satisfy the stopping criterion (77) versus the Reynolds number. We observe that $I t_{D N} \simeq 2 R e^{1 / 2}$.

Acknowledgements. We thank Prof. A. Valli for many fruitful discussions during the preparation of this report.

\section{REFERENCES}

[1] C. Bernardi and Y. Maday, Approximations spectrales de problèmes aux limites elliptiques. Springer-Verlag, Paris (1992).

[2] P. Bjørstad and O.B. Widlund, Iterative methods for the solution of elliptic problems on regions partitioned into substructures. SIAM J. Numer. Anal. 23 (1986) 1097-1120.

[3] L. Fatone, Homogeneous and heterogeneous models for incompressible flows. Ph.D. thesis, Università degli Studi di Milano (1999).

[4] L. Fatone, P. Gervasio and A. Quarteroni, Multimodels for incompressible flows. J. Math. Fluid Mech. 2 (2000) 126-150.

[5] M. Feistauer and C. Schwab, Coupling of an interior Navier-Stokes problem with an exterior Oseen problem. Technical Report Research 98-01, ETH, Zurich (1998).

[6] M. Feistauer and C. Schwab, On coupled problems for viscous flow in exterior domains. Math. Models Methods Appl. Sci. 8 (1998) 657-684.

[7] M. Feistauer and C. Schwab, Coupled problems for viscous incompressible flow in exterior domains. In Applied nonlinear analysis. Kluwer/Plenum, New York (1999) 97-116.

[8] L.I.G. Kovasznay, Laminar flow behind two-dimensional grid. Proc. Cambridge Phil. Soc. 44 (1948) 58-62.

[9] Y. Maday and A.T. Patera, Spectral element methods for the incompressible Navier-Stokes equations. In State-of-the-art surveys on computational mechanics. A.K. Noor and J. T. Oden Eds., The American Society of Mechanical Engineers, New York (1989).

[10] A. Quarteroni, G. Sacchi Landriani, and A. Valli, Coupling of viscous and inviscid incompressible Stokes equations. Numer. Math. 59 (1991) 831-859.

[11] A. Quarteroni and A. Valli, Theory and application of Steklov-Poincaré operators for boundary-value problems. In Applied and Industrial Mathematics, R. Spigler Ed., Kluwer Academic Publisher, Dordest (1991) 179-203.

[12] A. Quarteroni and A. Valli, Domain decomposition methods for partial differential equations. Oxford Science Publications, Oxford (1999). 
[13] K. Schenk and F.K. Hebeker, Coupling of two dimensional viscous and inviscid incompressible Stokes equations. Technical Report Preprint 93-68 (SFB 359), Heidelberg University (1993).

[14] R. Temam, Navier-Stokes equations. Theory and numerical analysis. 3rd edn., North-Holland, Amsterdam (1984).

[15] R. Temam, Navier-Stokes equations and nonlinear functional analysis. SIAM, Philadelphia (1988).

[16] H.A. van der Vorst, Bi-CGSTAB: a fast and smoothly converging variant of Bi-CG for the solution of nonsymmetric linear systems. SIAM J. Sci. Statist. Comput. 13 (1992) 631-644.

To access this journal online: www.edpsciences.org 